\title{
Finite-dimensional complex manifolds on commutative Banach algebras and continuous families of compact complex manifolds
}

https://doi.org/10.1515/coma-2019-0012

Received November 8, 2018; accepted May 20, 2019

\begin{abstract}
Let $\Gamma(M)$ be the set of all global continuous cross sections of a continuous family $M$ of compact complex manifolds on a compact Hausdorff space $X$. In this paper, we introduce a $C(X)$-manifold structure on $\Gamma(M)$. Especially, if $X$ is contractible, then $\Gamma(M)$ is a finite-dimensional $C(X)$-manifold. Here, $C(X)$ denotes the Banach algebra of all complex-valued continuous functions on $X$.
\end{abstract}

Keywords: topological deformation of complex analytic structures, infinite-dimensional manifold, commutative $C^{\star}$-algebra, Serre-Swan theorem

MSC: 19L99, 32Q99, 46J99, 58B99

\section{Introduction}

Precise definitions will be given in the following sections. However, we believe that there is no difficulty in reading this section if the reader knows the very basic concepts of complex analytic families and Banach algebras.

An $n$-dimensional complex manifold is a manifold by biholomorphic mappings between open sets of the finite direct product $\mathbb{C}^{n}$ of the complex number field. On the other hand, when $A$ is a commutative Banach algebra, Lorch [7] gave a definition that an $A$-valued function on an open set of $A$ is holomorphic. The definition of a holomorphic function by Lorch can be straightforwardly generalized to a Fréchet differentiable mapping from an open set of a Banach $A$-module to a Banach $A$-module. (The definition of a Banach $A$-module is given in Definition 2.4.) That is, it is said to be $A$-holomorphic, if the Fréchet derivatives are $A$-linear. Therefore, a manifold modeled on Banach $A$-modules is easily defined. We call it an $A$-manifold. The finite direct product $A^{n}$ is the most simple example of a Banach $A$-module. So, an $n$-dimensional $A$-manifold is defined. However, in my opinion, it seems that so many nontrivial examples were not known (including the case of 1-dimensional manifolds, that is, Riemann surfaces). It seems that only Riemann sphere is already known ([1]).

On the other hand, complex analytic families of compact complex manifolds are the subject of deformation theory starting with Kodaira-Spencer [6]. Interesting and relatively simple examples of complex analytic families are found in Kodaira [5] §2.3. Roughly speaking, a complex analytic family is a fiber bundle (without structure group) such that each fiber has a complex structure depending holomorphically on the corresponding point of the base space. We introduce a concept of compact continuous families of complex manifolds (Definition 1.3). Roughly speaking, a compact continuous family is a compact fiber bundle such that each

^Corresponding Author: Hiroki Yagisita: Department of Mathematics, Faculty of Science, Kyoto Sangyo University Motoyama, Kamigamo, Kita-ku, Kyoto-City, 603-8555, Japan 
fiber has a complex structure depending continuously on the corresponding point of the base space. (There may be a compact continuous family that is not a fiber bundle. This problem is interesting in the broader context, but less important in the context of this paper.) The family that restricts the base space of a complex analytic family to a compact subset becomes a compact continuous family (Remark of Definition 1.3). Therefore, there are many examples of compact continuous families.

If $X$ is a compact Hausdorff space, then the algebra $C(X)$ of all complex valued continuous functions on $X$ is the most basic example of a commutative Banach algebra (furthermore, a commutative $C^{\star}$-algebra). The set of all global continuous cross sections of an Hermitian vector bundle on $X$ is a Banach $C(X)$-module (Example 2.7). In this paper, we see that if the set of all global continuous cross sections of a compact continuous family $M$ on the base space $X$ is denoted by $\Gamma(M)$, then the structure of a $C(X)$-manifold modeled on the $C(X)$-modules of all global continuous cross sections of some Hermitian vector bundles on $X$ is introduced into $\Gamma(M)$ (Corollary 3.9). Therefore, especially, if $X$ is contractible, then $\Gamma(M)$ is a finite-dimensional $C(X)$-manifold (Corollary 3.10). Our main result (Theorem 3.6) is a little generalization of Corollary 3.9.

When $X$ is a differentiable manifold, Kobayashi [4] and Manoharan [8, 9, 10] constructed examples of $C^{\infty}(X ; \mathbb{R})$-manifolds. The basic idea of our proof is similar to them. However, the spray corresponding to LeviCivita connection (or the canonical connection) of an Hermitian manifold is only differentiable, and generally not holomorphic. Overcoming this difficulty is the technically major part in our proof.

In Sections 1 and 2, we give definitions of the basic concepts. In Section 3, we state our main result (Theorem 3.6) and a key proposition (Proposition 3.5) for the proof of the main result. In Section 4, we prove the key proposition.

\section{Compact continuous families of complex manifolds}

In this and the next sections, we introduce two basic concepts of this paper.

Definition 1.1 (Canonical real coordinate system) :

The mapping that maps a complex vector $z=x+\sqrt{-1} y \in \mathbb{C}^{n}$ to its real and imaginary pair $(x, y) \in \mathbb{R}^{2 n}$, i.e.,

$$
\begin{gathered}
\left(x_{1}+\sqrt{-1} y_{1}, x_{2}+\sqrt{-1} y_{2}, \cdots, x_{n}+\sqrt{-1} y_{n}\right) \\
\mapsto\left(\left(x_{1}, x_{2}, \cdots, x_{n}\right),\left(y_{1}, y_{2}, \cdots, y_{n}\right)\right)
\end{gathered}
$$

is called the canonical real coordinate system.

Hereafter, when we consider an open set of $\mathbb{C}^{n}$ to be a $C^{\infty}$-manifold, we use the canonical real coordinate system as its local coordinate system.

Definition 1.2 (Open polydisk) :

For $r>0$, let

$$
\begin{gathered}
D_{r}^{n}:=\left\{\left(z_{1}, z_{2}, \cdots, z_{n}\right) \in \mathbb{C}^{n}\left|\max _{k}\right| z_{k} \mid<r\right\} \\
=\left\{\left(\left(x_{1}, x_{2}, \cdots, x_{n}\right),\left(y_{1}, y_{2}, \cdots, y_{n}\right)\right) \in \mathbb{R}^{2 n} \mid\right. \\
\left.\max _{k} \sqrt{x_{k}^{2}+y_{k}^{2}}<r\right\} .
\end{gathered}
$$

Let $D^{n}:=D_{1}^{n}$. $D^{n}$ is called the unit open polydisk.

Definition 1.3 (Compact continuous family of complex manifolds) :

$M:=(M, X, \pi, S)$ is said to be a compact continuous family (of $n$-dimensional complex manifolds), if it satisfies the followings.

(1) $M$ and $X$ are compact Hausdorff spaces. $\pi$ is a continuous surjection form $M$ to $X$.

(2) $S$ is a non-empty set, which will be called the system of local trivialization coordinate neighborhoods of $M$ by Definition 1.4 below. For any $\varphi \in S, \varphi$ is a map from a non-empty open set $M_{\varphi}$ of $M$ to the unit open polydisk $D^{n}$. For $\varphi \in S$, let

$$
\left.\pi\right|_{\varphi}:=\pi_{\uparrow M_{\varphi}} .
$$


For any $\varphi \in S$, there exists a non-empty open set $X_{\varphi}$ of $X$ such that $\left(\varphi,\left.\pi\right|_{\varphi}\right)$ is a homeomorphism from $M_{\varphi}$ to $D^{n} \times X_{\varphi}$.

(3) For $\varphi \in S$ and $t \in X_{\varphi}$, let

$$
\begin{gathered}
M_{\varphi, t}:=M_{\varphi} \cap \pi^{-1}(\{t\}), \\
\left.\varphi\right|_{t}:=\varphi_{\left\lceil M_{\varphi, t}\right.} .
\end{gathered}
$$

For any $\varphi_{1}, \varphi_{2} \in S$ and $t \in X_{\varphi_{1}} \cap X_{\varphi_{2}}$, the coordinate transformation

$$
\varphi_{2}\left|t \circ \varphi_{1}\right|_{t}^{-1}: \varphi_{1}\left(M_{\varphi_{1}, t} \cap M_{\varphi_{2}, t}\right) \rightarrow \varphi_{2}\left(M_{\varphi_{1}, t} \cap M_{\varphi_{2}, t}\right)
$$

is holomorphic.

(4) $\quad M=\cup_{\varphi \in S} M_{\varphi}$ holds.

Remark :

(1) Each fiber of a compact continuous family is a compact complex manifold. Because $\pi$ is a continuous surjection, the assumption that $X$ is compact is actually redundant.

(2) Suppose that $(M, B, \pi)$ is a complex analytic family of compact complex manifolds. Then, by the usual argument (e.g., immediately after [5] Definition 2.8), we see that for any non-empty compact subset $X$ of $B, \pi^{-1}(X)$ is a compact continuous family. We also note that a complex analytic family is a fiber bundle without structure group ([5] Theorem 2.5).

Unlike complex analytic families, it seems that continuous families are not readily available in the literature. Therefore, we have to confirm basic facts of compact continuous families on our own.

Definition 1.4 (Local trivialization coordinate neighborhood) :

When $M:=(M, X, \pi, S)$ is a compact continuous family, $M$ is called the total space, $X$ is called the base space, $\pi$ is called the projection and $S$ is called the system of local trivialization coordinate neighborhoods.

Since the total space of a compact continuous family is compact, the system of local trivialization coordinate neighborhoods can be assumed to be a finite set.

Definition 1.5 (Finite continuous family) :

A compact continuous family whose system of local trivialization coordinate neighborhoods is a finite set is called a finite continuous family.

In order to show the existence of a suitable system of bump functions in our structure, we define the following.

Definition 1.6 (Compact coordinate neighborhood) :

Let $(M, X, \pi, S)$ be a finite continuous family. Then, $\left(\left\{\left(M_{\varphi}^{\prime}, X_{\varphi}^{\prime}\right)\right\}_{\varphi \in S}, r_{0}\right)$ is said to be a system of compact coordinate neighborhoods of $M$, if it satisfies the followings.

(1) $M_{\varphi}^{\prime}$ is an open set of $M, X_{\varphi}^{\prime}$ is an open set of $X$ and

$$
\overline{M_{\varphi}^{\prime}} \subset M_{\varphi}, \overline{X_{\varphi}^{\prime}} \subset X_{\varphi}
$$

hold.

(2) $r_{0} \in(0,1)$ holds.

(3) The homeomorphism $\left(\varphi,\left.\pi\right|_{\varphi}\right)$ from $M_{\varphi}$ to $D^{n} \times X_{\varphi}$ maps $M_{\varphi}^{\prime}$ onto $D_{r_{0}}^{n} \times X_{\varphi}^{\prime}$. That is,

$$
M_{\varphi}^{\prime}=(\varphi, \pi \mid \varphi)^{-1}\left(D_{r_{0}}^{n} \times X_{\varphi}^{\prime}\right)
$$

holds.

(4) $\quad M=\cup_{\varphi \in S} M_{\varphi}^{\prime}$ holds.

Remark : In the above definition, neither $M_{\varphi}^{\prime} \neq \emptyset$ nor $X_{\varphi}^{\prime} \neq \emptyset$ is required.

Definition 1.7 (Bump system) :

Let $(M, X, \pi, S)$ be a finite continuous family. Then, $\left(\left\{\left(\rho_{\varphi}, M_{\varphi}^{\prime}, X_{\varphi}^{\prime}\right)\right\}_{\varphi \in S}, r_{0}\right)$ is said to be a bump system of $M$, if it satisfies the followings.

(1) $\left(\left\{\left(M_{\varphi}^{\prime}, X_{\varphi}^{\prime}\right)\right\}_{\varphi \in S}, r_{0}\right)$ is a system of compact coordinate neighborhoods of $M$. 
(2) $\rho_{\varphi}$ is a continuous function from $M$ to $[0,+\infty)$ and

$$
\operatorname{supp}\left(\rho_{\varphi}\right) \subset M_{\varphi}^{\prime}
$$

holds.

(3) The function

$$
\rho_{\varphi} \circ\left(\varphi,\left.\pi\right|_{\varphi}\right)^{-1}: D^{n} \times X_{\varphi} \rightarrow[0,+\infty)
$$

is $C^{\infty}$-class with respect to the variable $(x, y) \in D^{n}\left(\subset \mathbb{R}^{2 n}\right)$. For any multi-indexes $\alpha$ and $\beta$, the function

$$
\partial_{x}{ }^{\alpha} \partial_{y}{ }^{\beta}\left(\rho_{\varphi} \circ\left(\varphi,\left.\pi\right|_{\varphi}\right)^{-1}\right): D^{n} \times X_{\varphi} \rightarrow \mathbb{R}
$$

is continuous.

(4) For any $p \in M$, there exists $\varphi \in S$ such that

$$
\rho_{\varphi}(p) \neq 0
$$

holds.

Remark : In the above definition, $\sum_{\varphi \in S} \rho_{\varphi}=1$ is not required.

Lemma 1.8 (Existence of a bump system) :

Suppose that $M$ is a finite continuous family. Then, there exists a bump system of $M$.

Proof: $M$ is normal and $\left\{M_{\varphi}\right\}_{\varphi \in S}$ is a finite open covering of $M$. Hence, there exists an open covering $\left\{G_{\varphi}\right\}_{\varphi \in S}$ of $M$ such that for any $\varphi \in S$,

$$
\overline{G_{\varphi}} \subset M_{\varphi}
$$

holds. For $\varphi \in S$, we set

$$
X_{\varphi}^{\prime}:=\pi\left(G_{\varphi}\right) .
$$

Then,

$$
\overline{X_{\varphi}^{\prime}}=\overline{\pi\left(G_{\varphi}\right)}=\pi\left(\overline{G_{\varphi}}\right) \subset \pi\left(M_{\varphi}\right)=X_{\varphi}
$$

holds. In addition, since $G_{\varphi}$ is an open set of $M_{\varphi}, X_{\varphi}^{\prime}$ is an open set of $X_{\varphi}$. So, $X_{\varphi}^{\prime}$ is an open set of $X$. On the other hand, since $\cup_{\varphi \in S} \varphi\left(\overline{G_{\varphi}}\right)$ is a compact subset of $D^{n}\left(:=D_{1}^{n}\right)$, there exists $r_{0} \in(0,1)$ such that

$$
\cup_{\varphi \in S} \varphi\left(\overline{G_{\varphi}}\right) \subset D_{r_{0}}^{n}
$$

holds. Now, we set

$$
M_{\varphi}^{\prime}:=\left(\varphi,\left.\pi\right|_{\varphi}\right)^{-1}\left(D_{r_{0}}^{n} \times X_{\varphi}^{\prime}\right) .
$$

$M_{\varphi}^{\prime}$ is an open set of $M$. Also, as $\overline{D_{r_{0}}^{n}} \times \overline{X_{\varphi}^{\prime}}$ is a compact subset of $D^{n} \times X_{\varphi}$,

$$
\overline{M_{\varphi}^{\prime}}=\left(\varphi,\left.\pi\right|_{\varphi}\right)^{-1}\left(\overline{D_{r_{0}}^{n}} \times \overline{X_{\varphi}^{\prime}}\right) \subset M_{\varphi}
$$

holds. From

$$
\left(\varphi,\left.\pi\right|_{\varphi}\right)\left(G_{\varphi}\right) \subset \varphi\left(G_{\varphi}\right) \times \pi\left(G_{\varphi}\right) \subset D_{r_{0}}^{n} \times X_{\varphi}^{\prime},
$$

$G_{\varphi} \subset M_{\varphi}^{\prime}$ holds. Therefore, $M=\cup_{\varphi \in S} G_{\varphi}=\cup_{\varphi \in S} M_{\varphi}^{\prime}$ holds. $\left(\left\{\left(M_{\varphi}^{\prime}, X_{\varphi}^{\prime}\right)\right\}_{\varphi \in S}, r_{0}\right)$ is a system of compact coordinate neighborhoods of $M$.

Similarly, there exist $\left\{\left(M_{\varphi}^{\prime \prime}, X_{\varphi}^{\prime \prime}\right)\right\}_{\varphi \in S}$ and $r_{1} \in\left(0, r_{0}\right)$ such that $M_{\varphi}^{\prime \prime}$ is an open set of $M, X_{\varphi}^{\prime \prime}$ is an open set of $X$ and

$$
\begin{gathered}
\overline{M_{\varphi}^{\prime \prime}} \subset M_{\varphi}^{\prime}, \quad \overline{X_{\varphi}^{\prime \prime}} \subset X_{\varphi}^{\prime}, \\
M_{\varphi}^{\prime \prime}=\left(\varphi,\left.\pi\right|_{\varphi}\right)^{-1}\left(D_{r_{1}}^{n} \times X_{\varphi}^{\prime \prime}\right), \\
M=\cup_{\varphi \in S} M_{\varphi}^{\prime \prime}
\end{gathered}
$$

hold. Further, similarly, there exist $\left\{\left(M_{\varphi}^{\prime \prime \prime}, X_{\varphi}^{\prime \prime \prime}\right)\right\}_{\varphi \in S}$ and $r_{2} \in\left(0, r_{1}\right)$ such that $M_{\varphi}^{\prime \prime \prime}$ is an open set of $M, X_{\varphi}^{\prime \prime \prime}$ is an open set of $X$ and

$$
\overline{M_{\varphi}^{\prime \prime \prime}} \subset M_{\varphi}^{\prime \prime}, \overline{X_{\varphi}^{\prime \prime \prime}} \subset X_{\varphi}^{\prime \prime},
$$




$$
\begin{gathered}
M_{\varphi}^{\prime \prime \prime}=\left(\varphi,\left.\pi\right|_{\varphi}\right)^{-1}\left(D_{r_{2}}^{n} \times X_{\varphi}^{\prime \prime \prime}\right), \\
M=\cup_{\varphi \in S} M_{\varphi}^{\prime \prime \prime}
\end{gathered}
$$

hold.

Now, since $X$ is normal, for $\varphi \in S$, there exists a non-negative continuous function $\rho_{1, \varphi}$ on $X$ such that

$$
\overline{X_{\varphi}^{\prime \prime \prime}} \subset\left\{t \in X \mid \rho_{1, \varphi}(t) \neq 0\right\} \subset X_{\varphi}^{\prime \prime}
$$

holds. Also, there exists a non-negative $C^{\infty}$-function $\rho_{2}$ on $\mathbb{C}\left(=\mathbb{C}^{1}=\mathbb{R}^{2}\right)$ such that

$$
\left\{(a, b) \in \mathbb{R}^{2} \mid \rho_{2}(a, b) \neq 0\right\}=D_{r_{1}}^{1}
$$

holds. Now, we define a non-negative function $\rho_{\varphi}$ on $M$ as

$$
\rho_{\varphi}(p):=\left\{\begin{array}{cr}
\rho_{1, \varphi}(\pi(p))\left(\prod_{k=1,2, \cdots, n} \rho_{2}\left(\varphi_{(k)}(p)\right)\right) & \left(p \in M_{\varphi}\right), \\
0 & \left(p \in M \backslash M_{\varphi}\right) .
\end{array}\right.
$$

Here, $\varphi_{(k)}$ is the $k$-th component of $\varphi$. Now, because of

$$
\left\{p \in M \mid \rho_{\varphi}(p) \neq 0\right\} \subset\left\{p \in M_{\varphi} \mid\left(\varphi,\left.\pi\right|_{\varphi}\right)(p) \in D_{r_{1}}^{n} \times X_{\varphi}^{\prime \prime}\right\}=M_{\varphi}^{\prime \prime},
$$

$\rho_{\varphi}$ is a continuous function such that

$$
\operatorname{supp}\left(\rho_{\varphi}\right) \subset \overline{M_{\varphi}^{\prime \prime}} \subset M_{\varphi}^{\prime}
$$

holds. However, because

$$
M_{\varphi}^{\prime \prime \prime}=\left\{p \in M_{\varphi} \mid\left(\varphi,\left.\pi\right|_{\varphi}\right)(p) \in D_{r_{2}}^{n} \times X_{\varphi}^{\prime \prime \prime}\right\} \subset\left\{p \in M \mid \rho_{\varphi}(p) \neq 0\right\}
$$

also holds,

$$
M=\cup_{\varphi \in S} M_{\varphi}^{\prime \prime \prime}=\cup_{\varphi \in S}\left\{p \in M \mid \rho_{\varphi}(p) \neq 0\right\}
$$

holds.

Definition 1.9 (Continuous family with bumps) :

$M:=\left(M, X, \pi, S,\left\{\left(\rho_{\varphi}, M_{\varphi}^{\prime}, X_{\varphi}^{\prime}\right)\right\}_{\varphi \in S}, r_{0}\right)$ is said to be a continuous family with bumps, if $(M, X, \pi, S)$ is a finite continuous family and $\left(\left\{\left(\rho_{\varphi}, M_{\varphi}^{\prime}, X_{\varphi}^{\prime}\right)\right\}_{\varphi \in S}, r_{0}\right)$ is a bump system of $M$.

Hereafter, the continuous family $M\left(=\left(M, X, \pi, S,\left\{\left(\rho_{\varphi}, M_{\varphi}^{\prime}, X_{\varphi}^{\prime}\right)\right\}_{\varphi \in S}, r_{0}\right)\right)$ with bumps is fixed.

Definition 1.10 (Real tangent bundle) :

Let $N$ be an $n$-dimensional complex manifold. Then, for $q \in N$, its real tangent space $\left(T_{\mathbb{R}}\right)_{q}(N)$ is a complex linear space by the almost complex structure of $N$. That is, when $z=x+\sqrt{-1} y$ is a holomorphic local coordinate system of $N$, set

$$
\sqrt{-1}\left(\frac{\partial}{\partial x_{k}}\right)_{q}:=\left(\frac{\partial}{\partial y_{k}}\right)_{q}, \quad \sqrt{-1}\left(\frac{\partial}{\partial y_{k}}\right)_{q}:=-\left(\frac{\partial}{\partial x_{k}}\right)_{q} .
$$

In addition, set

$$
T_{\mathbb{R}}(N):=\cup_{q \in N}\left(T_{\mathbb{R}}\right)_{q}(N) .
$$

$T_{\mathbb{R}}(N)$ is a holomorphic vector bundle on $N$.

Remark : The real tangent bundle $T_{\mathbb{R}}(N)$ is holomorphically isomorphic to the holomorphic tangent bundle $T^{\prime}(N)$ by

$$
\left(\frac{\partial}{\partial x_{k}}\right)_{q} \in T_{\mathbb{R}}(N) \mapsto\left(\frac{\partial}{\partial z_{k}}\right)_{q}:=\frac{1}{2}\left(\left(\frac{\partial}{\partial x_{k}}\right)_{q}-\sqrt{-1}\left(\frac{\partial}{\partial y_{k}}\right)_{q}\right) \in T^{\prime}(N) .
$$

Lemma 1.11 : 
Let $T$ be a topological space. Let $U$ be an open set of $\mathbb{C}^{n} \times T$. Suppose that $f$ is a complex valued continuous function on $U$ and for any $t \in T$, the map $z \mapsto f(z, t)$ is holomorphic. Then, for any multi-index $\gamma$,

$$
\partial_{z}^{\gamma} f: U \rightarrow \mathbb{C}
$$

is continuous.

Proof : From the induction, it suffices to show it in the case of $|\gamma|=1$. Furthermore, when $|\gamma|=1$ holds, even if we assume $n=1$, we do not lose generality. Let $n=1$.

Let $(w, s) \in U$ and $\varepsilon>0$. Then, there exist $r>0$ and an open set $T^{\prime}$ of $T$ such that $s \in T^{\prime}$ and

$$
|z-w|<2 r, t \in T^{\prime} \quad \Rightarrow \quad(z, t) \in U
$$

hold. Further, for any $\tau \in[0,2 \pi]$, there exist $\delta_{\tau} \in(0, r)$ and an open set $T_{\tau}$ of $T^{\prime}$ such that $s \in T_{\tau}$ and

$$
\begin{gathered}
|\theta-\tau|<\delta_{\tau},|z-w|<\delta_{\tau}, t \in T_{\tau} \\
\Rightarrow \\
\left|f\left(z+r e^{\sqrt{-1} \theta}, t\right)-f\left(w+r e^{\sqrt{-1} \tau}, s\right)\right|<\frac{1}{2} r \varepsilon
\end{gathered}
$$

hold. There exists a finite subset $K(\neq \emptyset)$ of $[0,2 \pi]$ such that

$$
\theta \in[0,2 \pi] \Rightarrow \exists \tau \in K:|\theta-\tau|<\delta_{\tau}
$$

holds. Now, we set

$$
\delta:=\min _{\tau \in K} \delta_{\tau}, \quad T^{\prime \prime}:=\cap_{\tau \in K} T_{\tau}
$$

$\delta>0$ and $s \in T^{\prime \prime}$ hold. $T^{\prime \prime}$ is an open set of $T$.

Now, let $|z-w|<\delta$ and $t \in T^{\prime \prime}$. Then, for any $\theta \in[0,2 \pi]$, there exists $\tau \in K$ such that

$$
\begin{gathered}
\left|f\left(z+r e^{\sqrt{-1} \theta}, t\right)-f\left(w+r e^{\sqrt{-1} \theta}, s\right)\right| \\
\leq\left|f\left(z+r e^{\sqrt{-1} \theta}, t\right)-f\left(w+r e^{\sqrt{-1} \tau}, s\right)\right|+\left|f\left(w+r e^{\sqrt{-1} \tau}, s\right)-f\left(w+r e^{\sqrt{-1} \theta}, s\right)\right| \\
<r \varepsilon
\end{gathered}
$$

holds. So,

$$
\begin{aligned}
& \left|\left(\partial_{z} f\right)(z, t)-\left(\partial_{z} f\right)(w, s)\right| \\
& =\left|\frac{1}{2 \pi r} \int_{\theta \in[0,2 \pi]} e^{-\sqrt{-1} \theta}\left(f\left(z+r e^{\sqrt{-1} \theta}, t\right)-f\left(w+r e^{\sqrt{-1} \theta}, s\right)\right) d \theta\right| \\
& <\varepsilon
\end{aligned}
$$

holds.

Notation : For $p \in M$, we introduce the notation

$$
F_{p}:=\pi^{-1}(\{\pi(p)\}) .
$$

That is, $F_{p}$ denotes the fiber through $p$.

Definition 1.12 (Trivial Hermitian metric) :

Let $\varphi \in S$. For $p \in M_{\varphi}$, define a complex inner product on $\left(T_{\mathbb{R}}\right)_{p}\left(F_{p}\right)$ as

$$
\left\langle\dot{p}_{1}, \dot{p}_{2}\right\rangle_{\varphi, p}:=\left\langle\left(D\left(\left.\varphi\right|_{\pi(p)}\right)\right)_{p}\left(\dot{p}_{1}\right),\left(D\left(\left.\varphi\right|_{\pi(p)}\right)\right)_{p}\left(\dot{p}_{2}\right)\right\rangle_{\mathbb{C}^{n}}
$$

Here, $D\left(\left.\varphi\right|_{\pi(p)}\right)$ is the differential of the holomorphic local coordinate system

$$
\left.\varphi\right|_{\pi(p)}:=\varphi_{\left\lceil M_{\varphi, \pi(p)}\right.}=\varphi_{\left\lceil M_{\varphi} \cap F_{p}\right.}
$$


of $F_{p}$ and $\langle\cdot, \cdot\rangle_{\mathbb{C}^{n}}$ is the canonical inner product (the canonical Hermitian metric) on $\mathbb{C}^{n}$. Also, $\|\cdot\| \varphi, p$ denotes the norm by this complex inner product. That is, let

$$
\|\dot{p}\|_{\varphi, p}:=\sqrt{\langle\dot{p}, \dot{p}\rangle_{\varphi, p}} .
$$

For $t \in X_{\varphi}$, by the complex inner products, the complex manifold

$$
M_{\varphi, t}:=M_{\varphi} \cap \pi^{-1}(\{t\})
$$

is a Hermitian manifold that is isomorphic to $D^{n}$. In addition, the topological space

$$
T_{\mathbb{R}}\left(M_{\varphi}\right):=\cup_{p \in M_{\varphi}}\left(T_{\mathbb{R}}\right)_{p}\left(F_{p}\right)
$$

is a continuous Hermitian vector bundle on the topological space $M_{\varphi}$.

Definition 1.13 (Hermitian real tangent bundle) :

For $p \in M$, define a complex inner product on $\left(T_{\mathbb{R}}\right)_{p}\left(F_{p}\right)$ as

$$
\left\langle\dot{p}_{1}, \dot{p}_{2}\right\rangle_{p}:=\sum_{\varphi \in S} \rho_{\varphi}(p)\left\langle\dot{p}_{1}, \dot{p}_{2}\right\rangle_{\varphi, p} .
$$

Also, $\|\cdot\|_{p}$ denotes the norm by this complex inner product. That is, let

$$
\|\dot{p}\|_{p}:=\sqrt{\langle\dot{p}, \dot{p}\rangle_{p}} .
$$

For $t \in X$, by the complex inner products, the fiber $\pi^{-1}(\{t\})$ of the continuous family $M$ is a Hermitian manifold. In addition, the topological space

$$
T_{\mathbb{R}}(M):=\cup_{p \in M}\left(T_{\mathbb{R}}\right)_{p}\left(F_{p}\right)
$$

is a continuous Hermitian vector bundle on the total space $M$ of the continuous family. The projection $T_{\mathbb{R}}(M) \rightarrow M$ of the vector bundle is denoted by $\varpi$. That is, for $\dot{p} \in T_{\mathbb{R}}(M)$,

$$
\varpi(\dot{p}) \in M, \quad \dot{p} \in\left(T_{\mathbb{R}}\right)_{\varpi(\dot{p})}\left(F_{\varpi(\dot{p})}\right)
$$

hold. $\varpi(\dot{p})$ is called the base point of a real tangent vector $\dot{p}$.

Remark : In the definition of $T_{\mathbb{R}}(M)$, Lemma 1.11 was used. That is, according to Lemma 1.11, for any local trivialization coordinate systems $\psi_{1}$ and $\psi_{2}$ of $M$ that are compatible with each other, the local trivialization coordinate system of $T_{\mathbb{R}}(M)$ determined by $\psi_{1}$ and the one determined by $\psi_{2}$ are compatible with each other.

$$
-
$$

Lemma 1.14 :

Let $\varphi \in S$. Let $K$ be a compact subset of $M_{\varphi}$. Then, there exist an open set $U$ of $M_{\varphi}$ and $C>0$ such that the followings hold.

(1) $K \subset U$ holds.

(2) For any $p \in U$ and $\dot{p} \in\left(T_{\mathbb{R}}\right)_{p}\left(F_{p}\right)$,

$$
\|\dot{p}\|_{\varphi, p} \leq C\|\dot{p}\|_{p}, \quad\|\dot{p}\|_{p} \leq C\|\dot{p}\|_{\varphi, p}
$$

hold.

Proof : Both the Hermitian metrics are continuous. So, it follows.

Definition 1.15 (Distance on a fiber) :

Let $t \in X$. For a piecewise $C^{\infty}$-curve $c:[a, b] \rightarrow \pi^{-1}(\{t\})$, let

$$
L_{t}(c):=\int_{a}^{b}\left\|\frac{d}{d s}(c(s))\right\|_{c(s)} d s .
$$

For $p, q \in \pi^{-1}(\{t\})$, let

$$
d_{t}(p, q)
$$


$:=\inf \left(\left\{L_{t}(c) \mid c\right.\right.$ is a piecewise $C^{\infty}$-curve

with the start point $p$ and the end point $q\} \cup\{1\}$ ).

By $d_{t}, \pi^{-1}(\{t\})$ is a metric space.

Remark : $\operatorname{Re}\left(\langle z, w\rangle_{\mathbb{C}^{n}}\right)=\langle z, w\rangle_{\mathbb{R}^{2 n}}$ and especially, $\|z\|_{\mathbb{C}^{n}}=\|z\|_{\mathbb{R}^{2 n}}$ hold. Therefore, the norm defined by a complex inner product is the norm defined by a real one.

Lemma 1.16 :

Let $\varphi \in S$. Let $N$ be an open set of $M_{\varphi}$. Let $K$ be a compact subset of $N$. Then, there exist an open set $U$ of $N, \delta>0$ and $C>0$ such that the followings hold.

(1) $K \subset U$ holds.

(2) Suppose $p \in U$ and $q \in F_{p}$. Then,

$$
\begin{gathered}
d_{\pi(p)}(q, p)<\delta \\
\Rightarrow \\
q \in N,\|\varphi(q)-\varphi(p)\|_{\mathbb{C}^{n}} \leq C d_{\pi(p)}(q, p)
\end{gathered}
$$

holds.

Proof : From Lemma 1.14, there exist an open set $U^{\prime}$ of $M_{\varphi}$ and $C>0$ such that the followings hold.

[ (1) $K \subset U^{\prime}$ holds. (2) For any $p \in U^{\prime}$ and $\dot{p} \in\left(T_{\mathbb{R}}\right)_{p}\left(F_{p}\right)$, $\|\dot{p}\|_{\varphi, p} \leq C\|\dot{p}\|_{p}$ holds. ]

Then, $K \subset U^{\prime} \cap N$ holds, $U^{\prime} \cap N$ is an open set of $M$ and $K$ is a closed set of $M$. Because $M$ is normal, there exists an open set $U^{\prime \prime}$ of $M$ such that

$$
K \subset U^{\prime \prime} \subset \overline{U^{\prime \prime}} \subset U^{\prime} \cap N
$$

holds. Also, since $U^{\prime} \cap N \subset M_{\varphi}$ holds,

$$
K \subset U^{\prime \prime} \subset \overline{U^{\prime \prime}} \subset U^{\prime} \cap N \subset M_{\varphi}
$$

holds. So, for $p \in K$, there exist $\delta_{p}>0$ and an open set $T_{p}$ of $X$ such that

$$
\begin{gathered}
\pi(p) \in T_{p}, \\
\left\{z \in \mathbb{C}^{n} \mid\|z-\varphi(p)\|_{\mathbb{C}^{n}}<(C+1) \delta_{p}\right\} \times T_{p} \subset\left(\varphi,\left.\pi\right|_{\varphi}\right)\left(U^{\prime \prime}\right)
\end{gathered}
$$

hold. Then, there exists a finite subset $L$ of $K$ such that

$$
\left(\varphi,\left.\pi\right|_{\varphi}\right)(K) \subset \cup_{p \in L}\left(\left\{z \in \mathbb{C}^{n} \mid\|z-\varphi(p)\|_{\mathbb{C}^{n}}<\delta_{p}\right\} \times T_{p}\right)
$$

holds. Now, we set

$$
\begin{gathered}
\delta:=\min \left(\left\{\delta_{p}\right\}_{p \in L} \cup\{1\}\right), \\
V:=\cup_{p \in L}\left(\left\{z \in \mathbb{C}^{n} \mid\|z-\varphi(p)\|_{\mathbb{C}^{n}}<\delta_{p}\right\} \times T_{p}\right), \\
U:=\left(\varphi,\left.\pi\right|_{\varphi}\right)^{-1}(V) .
\end{gathered}
$$

$\delta \in(0,1]$ holds. $U$ is an open set of $N$. $U$ includes $K$.

Now, let $p \in U, q \in F_{p}$ and $d_{\pi(p)}(q, p)<\delta$. Then, there exists $p^{\prime} \in L$ such that

$$
\left\|\varphi(p)-\varphi\left(p^{\prime}\right)\right\|_{\mathbb{C}^{n}}<\delta_{p^{\prime}}, \quad \pi(p) \in T_{p^{\prime}}
$$

hold. So, for any $z \in \mathbb{C}^{n}$,

$$
\begin{gathered}
\|z-\varphi(p)\|_{\mathbb{C}^{n}}<C \delta \\
\Rightarrow \\
\left\|z-\varphi\left(p^{\prime}\right)\right\|_{\mathbb{C}^{n}} \leq\|z-\varphi(p)\|_{\mathbb{C}^{n}}+\left\|\varphi(p)-\varphi\left(p^{\prime}\right)\right\|_{\mathbb{C}^{n}}
\end{gathered}
$$




$$
\begin{gathered}
<C \delta+\delta_{p^{\prime}} \leq(C+1) \delta_{p^{\prime}} \\
\Rightarrow \\
(z, \pi(p)) \subset\left(\varphi,\left.\pi\right|_{\varphi}\right)\left(U^{\prime \prime}\right)
\end{gathered}
$$

holds. Therefore,

$$
\begin{aligned}
& \forall z \in \mathbb{C}^{n}:\left[\|z-\varphi(p)\|_{\mathbb{C}^{n}}<C \delta\right. \\
& \left.\Rightarrow\left[z \in D^{n},\left.\varphi\right|_{\pi(p)} ^{-1}(z) \in U^{\prime \prime}\right]\right]
\end{aligned}
$$

holds. Now, we set

$$
H:=\left\{z \in \mathbb{C}^{n} \mid\|z-\varphi(p)\|_{\mathbb{C}^{n}}<C \delta\right\} .
$$

$H$ is an open set of $D^{n}$. Further, we set

$$
\begin{gathered}
G:=\left.\varphi\right|_{\pi(p)} ^{-1}(H), \\
F:=F_{p} \backslash G .
\end{gathered}
$$

$G$ is an open set of $M_{\varphi, \pi(p)} . F$ is a closed set of $F_{p}$.

$$
p \in G \subset U^{\prime \prime}
$$

holds. Now, because of $d_{\pi(p)}(q, p)<\delta \leq 1$, there exists $\left\{c_{m}\right\}_{m \in \mathbb{N}}$ such that the followings hold.

[ (1) For any $m \in \mathbb{N}, c_{m}$ is a piecewise $C^{\infty}$-map from $[0,1]$ to $F_{p}$ and

$$
c_{m}(0)=p, \quad c_{m}(1)=q, \quad L_{\pi(p)}\left(c_{m}\right)<\delta
$$

hold. (2) $d_{\pi(p)}(q, p)=\lim _{m \rightarrow \infty} L_{\pi(p)}\left(c_{m}\right)$ holds. ]

Now, for $m \in \mathbb{N}$, we set

$$
s_{m}:=\min \left(c_{m}^{-1}(F) \cup\{1\}\right) .
$$

$s_{m} \in(0,1]$ holds. However, because of

$$
\begin{gathered}
s \in\left[0, s_{m}\right) \Rightarrow c_{m}(s) \in G, \\
c_{m}\left(\left[0, s_{m}\right]\right) \subset \bar{G} \subset \overline{U^{\prime \prime}} \subset U^{\prime} \cap N \subset M_{\varphi}
\end{gathered}
$$

holds. So, because of

$$
\begin{gathered}
\left\|\varphi\left(c_{m}\left(s_{m}\right)\right)-\varphi(p)\right\|_{\mathbb{C}^{n}} \leq \int_{0}^{s_{m}}\left\|\frac{d}{d s}\left(\left(\varphi \circ c_{m}\right)(s)\right)\right\|_{\mathbb{C}^{n}} d s \\
\leq \int_{0}^{s_{m}} C\left\|\frac{d}{d s}\left(c_{m}(s)\right)\right\|_{c_{m}(s)} d s=C L_{\pi(p)}\left(\left\{c_{m}(s)\right\}_{s \in\left[0, s_{m}\right]}\right)<C \delta,
\end{gathered}
$$

$\varphi\left(c_{m}\left(s_{m}\right)\right) \in H$ holds. Hence, $c_{m}\left(s_{m}\right) \in G$ and $s_{m}=1$ hold. Therefore,

$$
\begin{gathered}
q=c_{1}(1)=c_{1}\left(s_{1}\right) \in N, \\
\|\varphi(q)-\varphi(p)\|_{\mathbb{C}^{n}} \\
=\lim _{m \rightarrow \infty}\left\|\varphi\left(c_{m}(1)\right)-\varphi(p)\right\|_{\mathbb{C}^{n}} \\
=\lim _{m \rightarrow \infty}\left\|\varphi\left(c_{m}\left(s_{m}\right)\right)-\varphi(p)\right\|_{\mathbb{C}^{n}} \\
\leq \lim _{m \rightarrow \infty} C L_{\pi(p)}\left(\left\{c_{m}(s)\right\}_{s \in\left[0, s_{m}\right]}\right) \\
=\lim _{m \rightarrow \infty} C L_{\pi(p)}\left(c_{m}\right)=C d_{\pi(p)}(q, p)
\end{gathered}
$$

hold. 


\section{Remark on Lemma 1.16 :}

Let $\varphi \in S$. Let $N$ be an open set of $M_{\varphi}$. Let $K$ be a compact subset of $N$. Then, there exist an open set $U$ of $N, \delta>0$ and $C>0$ such that the followings hold.

(1) $K \subset U$ holds.

(2) Suppose that $p \in U$ and $q \in M_{\varphi, \pi(p)}\left(:=M_{\varphi} \cap F_{p}\right)$. Then,

$$
\begin{gathered}
\|\varphi(q)-\varphi(p)\|_{\mathbb{C}^{n}}<\delta \\
\Rightarrow \\
q \in N, \quad d_{\pi(p)}(q, p) \leq C\|\varphi(q)-\varphi(p)\|_{\mathbb{C}^{n}}
\end{gathered}
$$

holds. (This remark is not used.)

Proof : From Lemma 1.14, there exist an open set $U^{\prime}$ of $M_{\varphi}$ and $C>0$ such that the followings hold.

[ (1) $K \subset U^{\prime}$ holds. (2) For any $p \in U^{\prime}$ and $\dot{p} \in\left(T_{\mathbb{R}}\right)_{p}\left(F_{p}\right),\|\dot{p}\|_{p} \leq C\|\dot{p}\|_{\varphi, p}$ holds. ]

Then, $U^{\prime} \cap N$ is an open set of $M_{\varphi}$. $U^{\prime} \cap N$ includes $K$. So, for $p \in K$, there exist $\delta_{p}>0$ and an open set $T_{p}$ of $X$ such that

$$
\begin{gathered}
\pi(p) \in T_{p}, \\
\left\{z \in \mathbb{C}^{n} \mid\|z-\varphi(p)\|_{\mathbb{C}^{n}}<2 \delta_{p}\right\} \times T_{p} \subset\left(\varphi,\left.\pi\right|_{\varphi}\right)\left(U^{\prime} \cap N\right)
\end{gathered}
$$

hold. There exists a finite subset $L$ of $K$ such that

$$
\left(\varphi,\left.\pi\right|_{\varphi}\right)(K) \subset \cup_{p \in L}\left(\left\{z \in \mathbb{C}^{n} \mid\|z-\varphi(p)\|_{\mathbb{C}^{n}}<\delta_{p}\right\} \times T_{p}\right)
$$

holds. Now, we set

$$
\begin{gathered}
\delta:=\min \left(\left\{\delta_{p}\right\}_{p \in L} \cup\{1\}\right), \\
V:=\cup_{p \in L}\left(\left\{z \in \mathbb{C}^{n} \mid\|z-\varphi(p)\|_{\mathbb{C}^{n}}<\delta_{p}\right\} \times T_{p}\right), \\
U:=\left(\varphi,\left.\pi\right|_{\varphi}\right)^{-1}(V) .
\end{gathered}
$$

$\delta>0$ holds. $U$ is an open set of $N$. $U$ includes $K$.

Now, let $p \in U, q \in M_{\varphi, \pi(p)}$ and $\|\varphi(q)-\varphi(p)\|_{\mathbb{C}^{n}}<\delta$. For $s \in[0,1]$, we set

$$
c(s):=(1-s) \varphi(p)+s \varphi(q)=\varphi(p)+s(\varphi(q)-\varphi(p)) .
$$

Also, there exists $p^{\prime} \in L$ such that

$$
\left\|\varphi(p)-\varphi\left(p^{\prime}\right)\right\|_{\mathbb{C}^{n}}<\delta_{p^{\prime}}, \quad \pi(p) \in T_{p^{\prime}}
$$

hold. Hence, because of

$$
\begin{gathered}
\left\|c(s)-\varphi\left(p^{\prime}\right)\right\|_{\mathbb{C}^{n}} \leq\left\|\varphi(p)-\varphi\left(p^{\prime}\right)\right\|_{\mathbb{C}^{n}}+s\|\varphi(q)-\varphi(p)\|_{\mathbb{C}^{n}} \\
<\delta_{p^{\prime}}+s \delta \leq 2 \delta_{p^{\prime}} \\
(c(s), \pi(p)) \in\left(\varphi,\left.\pi\right|_{\varphi}\right)\left(U^{\prime} \cap N\right)
\end{gathered}
$$

holds. In particular, $q=\left(\varphi,\left.\pi\right|_{\varphi}\right)^{-1}(c(1), \pi(p)) \in N$ holds. Further, because of $\left(\varphi,\left.\pi\right|_{\varphi}\right)^{-1}(c(s), \pi(p)) \in U^{\prime}$,

$$
\begin{gathered}
d_{\pi(p)}(q, p) \leq L_{\pi(p)}\left(\left\{(\varphi, \pi \mid \varphi)^{-1}(c(s), \pi(p))\right\}_{s \in[0,1]}\right) \\
\quad \leq \int_{0}^{1} C\|\varphi(q)-\varphi(p)\|_{\mathbb{C}^{n}} d s=C\|\varphi(q)-\varphi(p)\|_{\mathbb{C}^{n}}
\end{gathered}
$$

holds. 
Definition 1.17 (Continuous section) :

Let $T$ be a non-empty subset of $X$. A continuous map $u$ from $T$ to $M$ is said to be a continuous section of $M$ on $T$, if for any $t \in T, \pi(u(t))=t$ holds. The set of all continuous sections of $M$ on $T$ is denoted by $\Gamma(M \mid T)$.

Definition 1.18 (Distance between continuous sections) :

Let $T$ be a non-empty subset of $X$. For $u, v \in \Gamma(M \mid T)$, let

$$
d_{T}(u, v):=\sup _{t \in T} d_{t}(u(t), v(t))
$$

By $d_{T}, \Gamma(M \mid T)$ is a metric space.

\section{Manifolds on commutative Banach algebras}

Definition 2.1 (Commutative algebra) :

$A$ is said to be a commutative algebra, if $A$ is a commutative ring with the multiplicative unit $1_{A}\left(\neq 0_{A}\right)$, it is a complex linear space and it satisfies

$$
\left(c 1_{A}\right) f=c f \quad(c \in \mathbb{C}, f \in A) .
$$

Remark : When $A$ is a commutative algebra, $\mathbb{C} \subset A$ holds.

Definition 2.2 (Commutative Banach algebra) :

$A$ is said to be a commutative Banach algebra, if $A$ is a commutative algebra, it is a complex Banach space and it satisfies

$$
\begin{gathered}
\|f g\| \leq\|f\|\|g\| \quad(f, g \in A), \\
\left\|1_{A}\right\|=1 .
\end{gathered}
$$

\section{Example 2.3 :}

Let $T$ be a non-empty topological space. Let $C_{b}(T)$ be the set of all complex valued bounded continuous functions on $T$. Let

$$
\|f\|:=\sup _{t \in T}|f(t)| \quad\left(f \in C_{b}(T)\right) .
$$

$C_{b}(T)$ is a commutative Banach algebra.

Definition 2.4 (Banach module) :

Let $A$ be a commutative Banach algebra. $X$ is said to be a Banach $A$-module, if $X$ is an $A$-module, it is a complex Banach space and it satisfies

$$
\begin{gathered}
\left(c 1_{A}\right) u=c u \quad(c \in \mathbb{C}, u \in X), \\
\|f u\|_{X} \leq\|f\|_{A}\|u\|_{X} \quad(f \in A, u \in X) .
\end{gathered}
$$

\section{Example 2.5 :}

Let $A$ be a commutative Banach algebra. Let

$$
\left\|\left(f_{1}, f_{2}, \cdots, f_{n}\right)\right\|:=\max _{k}\left\|f_{k}\right\|_{A} \quad\left(f_{1}, f_{2}, \cdots, f_{n} \in A\right) .
$$

The finite direct product $A^{n}$ is a Banach $A$-module.

Example 2.6 :

Let $T$ be a non-empty topological space. Let $X$ be a complex Banach space. Let $C(T ; X)$ be the set of all $X$-valued continuous functions on $T$. Let

$$
\|u\|:=\sup _{t \in T}\|u(t)\|_{X} \quad(u \in C(T ; X)),
$$




$$
C_{b}(T ; X):=\{u \in C(T ; X) \mid\|u\|<+\infty\} .
$$

$C_{b}(T ; X)$ is a Banach $C_{b}(T)$-module.

\section{Example 2.7 :}

Let $T$ be a non-empty topological space. Let $E$ be a continuous Hermitian vector bundle on $T$. Let $\Gamma(E)$ be the set of all continuous sections of $E$ on $T$. Let

$$
\begin{gathered}
\|u\|:=\sup _{t \in T}\|u(t)\|_{t}=\sup _{t \in T} \sqrt{\langle u(t), u(t)\rangle_{t}} \quad(u \in \Gamma(E)), \\
\Gamma_{b}(E):=\{u \in \Gamma(E) \mid\|u\|<+\infty\} .
\end{gathered}
$$

$\Gamma_{b}(E)$ is a Banach $C_{b}(T)$-module.

Definition 2.8 (Linear mapping) :

Let $A$ be a ring. Let $X$ and $Y$ be $A$-modules. A mapping $F: X \rightarrow Y$ is said to be $A$-linear, if it satisfies

$$
\begin{gathered}
F(u+v)=F(u)+F(v) \quad(u, v \in X), \\
F(f u)=f F(u) \quad(f \in A, u \in X) .
\end{gathered}
$$

Remark : Let $X$ and $Y$ be Banach $A$-modules. If a mapping $F: X \rightarrow Y$ is $A$-linear, then $F$ is complex linear. Example 2.9 :

Let $T$ be a non-empty topological space. Let $A$ be a continuous complex linear map from a complex Banach space $X$ to a complex Banach space $Y$. If $\tilde{A}$ is defined as

$$
(\tilde{A}(u))(t):=A(u(t)) \quad\left(u \in C_{b}(T ; X), t \in T\right),
$$

then $\tilde{A}$ is a continuous $C_{b}(T)$-linear map from $C_{b}(T ; X)$ to $C_{b}(T ; Y)$.

Definition 2.10 (Fréchet derivative) :

Let $X$ and $Y$ be real Banach spaces. Let $f$ be a map from an open set $U$ of $X$ to $Y$.

(1) Let $p \in U$. Let $A: X \rightarrow Y$ be a continuous real linear map. $A$ is said to be the Fréchet derivative of $f$ at the point $p$, if for any $\varepsilon>0$, there exists $\delta>0$ such that

$$
\|h\|_{X} \leq \delta \Rightarrow\|f(p+h)-(f(p)+A h)\|_{Y} \leq \varepsilon\|h\|_{X}
$$

holds.

(2) $f$ is said to be Fréchet differentiable (on $U$ ), if for any $p \in U$, there exists Fréchet derivative of $f$ at the point $p$.

Remark : If $A$ and $B$ are Fréchet derivatives of $f$ at a point $p$, then $A=B$ holds. The Fréchet derivative of $f$ at a point $p$ is denoted by $f^{\prime}(p),(D f)(p)$ or $(D f)_{p}$.

Definition 2.11 (Complex differentiable) :

Let $X$ and $Y$ be complex Banach spaces. Let $f$ be a map from an open set $U$ of $X$ to $Y$. $f$ is said to be complex differentiable (on $U$ ), if $f$ is Fréchet differentiable and for any $p \in U$, the Fréchet derivative $(D f)_{p}$ is complex linear.

Remark (Complex power expansion) :

Suppose that $f$ is complex differentiable. Then, $f$ is complex analytic (that is, there exists the complex power expansion in a neighborhood of each point). In particular, $D f$ is complex differentiable. ([12] Theorem 14.7)

Let us generalize the definition of an $A$-differentiable function from an open set of a commutative Banach algebra $A$ to $A$ introduced by Lorch [7] to a mapping from an open set of a Banach $A$-module to a Banach $A$ module.

Definition 2.12 (Differentiable mapping) :

Let $X$ and $Y$ be Banach $A$-modules. Let $f$ be a mapping from an open set $U$ of $X$ to $Y$. $f$ is said to be $A$ differentiable (on $U$ ), if $f$ is Fréchet differentiable and for any $p \in U$, the Fréchet derivative $(D f)_{p}$ is $A$-linear. 
Remark : If a map is $A$-differentiable, then it is complex differentiable.

The following theorem is known about the Lorch differential of an $A$-valued function on an open set of $A$ ([3] §3.19 and §26.4). We do not use this theorem. However, with this, the identity of $C_{b}(T)$-differentiable mappings from open sets of $C_{b}(T)$ to $C_{b}(T)$ is almost obvious. Rather, it would be more accurate to say that this theorem is a generalization of that fact.

Theorem 2.13 (Taylor expansion) :

Let $A$ be a commutative Banach algebra. Let $c \in A$ and $r \in(0,+\infty]$. Let $D_{r}(c):=\{x \in A \mid\|x-c\|<r\}$.

(1) Let $\left\{a_{n}\right\}_{n=0}^{\infty}$ be a sequence of $A$. Suppose $r=\liminf _{n \rightarrow \infty}\left(\left\|a_{n}\right\|^{-\frac{1}{n}}\right)$. Then,

$$
x \mapsto \sum_{n=0}^{\infty} a_{n}(x-c)^{n}
$$

is an $A$-differentiable mapping from $D_{r}(c)$ to $A$.

(2) Suppose that $f$ is an $A$-differentiable mapping from $D_{r}(c)$ to $A$. Then, there uniquely exists a sequence $\left\{a_{n}\right\}_{n=0}^{\infty}$ of $A$ such that

$$
f(x)=\sum_{n=0}^{\infty} a_{n}(x-c)^{n}
$$

holds. Further, $r \leq \liminf _{n \rightarrow \infty}\left(\left\|a_{n}\right\|^{-\frac{1}{n}}\right)$ holds.

Definition 2.14 (Manifold on a commutative Banach algebra) :

Let $A$ be a commutative Banach algebra. Then, $M:=(M, S)$ is said to be a complex manifold on the commutative Banach algebra $A$ (or an $A$-manifold) with the system $S$ of coordinate neighborhoods, if the followings hold.

$M$ is a Hausdorff space. $S$ is a set. For any $\varphi \in S$, there exists a Banach $A$-module $X$ such that $\varphi$ is a homeomorphism from an open set of $M$ to an open set of $X$. For any $p \in M$, there exists $\varphi \in S$ such that $p$ belongs to the domain of $\varphi$. For any $\varphi_{1}, \varphi_{2} \in S$, the coordinate transformation

$$
\varphi_{2} \circ \varphi_{1}^{-1}: \varphi_{1}\left(U_{1} \cap U_{2}\right) \rightarrow \varphi_{2}\left(U_{1} \cap U_{2}\right)
$$

is $A$-differentiable. Here, $U_{1}$ and $U_{2}$ are the domains of $\varphi_{1}$ and $\varphi_{2}$, respectively.

\section{The main result}

First of all, recall that the continuous family $M\left(=\left(M, X, \pi, S,\left\{\left(\rho_{\varphi}, M_{\varphi}^{\prime}, X_{\varphi}^{\prime}\right)\right\}_{\varphi \in S}, r_{0}\right)\right)$ with bumps was fixed.

Definition 3.1 (Hermitian vector bundle induced by a continuous section) :

Let $T$ be a non-empty subset of $X$. Let $u \in \Gamma(M \mid T)$. Let

$$
\begin{gathered}
u^{*}\left(T_{\mathbb{R}}(M)\right):=T_{\mathbb{R}}(M) \mid u(T) \\
\quad=\cup_{p \in u(T)}\left(T_{\mathbb{R}}\right)_{p}\left(F_{p}\right) .
\end{gathered}
$$

Because $u$ is a homeomorphism from $T$ to $u(T), u^{\star}\left(T_{\mathbb{R}}(M)\right)$ is a continuous Hermitian vector bundle on the topological space $T$.

Definition 3.2 (Norm of a bounded continuous section) :

Let $T$ be a non-empty subset of $X$. Let $u \in \Gamma(M \mid T)$. Then, the norm of a bounded continuous section of $u^{\star}\left(T_{\mathbb{R}}(M)\right)$ on $T$ is denoted by $\|\cdot\|_{u}$. That is, for $\dot{u} \in \Gamma_{b}\left(u^{\star}\left(T_{\mathbb{R}}(M)\right)\right)$, let

$$
\|\dot{u}\|_{u}:=\sup _{t \in T}\|\dot{u}(t)\|_{u(t)} .
$$

$\Gamma_{b}\left(u^{\star}\left(T_{\mathbb{R}}(M)\right)\right)$ is a Banach $C_{b}(T)$-module. 
Definition 3.3 (Holomorphic normal coordinate neighborhood) :

Let $T$ be a non-empty subset of $X$. Let $u \in \Gamma(M \mid T)$. Then, $(U, \Psi)$ is said to be a holomorphic normal coordinate neighborhood of $u$, if it satisfies the followings.

(1) $U$ is an open set of $\pi^{-1}(T)$. For any $t \in T$,

$$
u(t) \in U_{t}\left(:=U \cap \pi^{-1}(\{t\})\right)
$$

holds.

(2) For $p \in M$, let

$$
B_{p}\left(T_{\mathbb{R}}\right):=\left\{\dot{p} \in\left(T_{\mathbb{R}}\right)_{p}\left(F_{p}\right) \mid\|\dot{p}\|_{p}<1\right\} .
$$

$\Psi$ is a homeomorphism from $U$ to $\cup_{p \in u(T)} B_{p}\left(T_{\mathbb{R}}\right)$. For any $t \in T,\left.\Psi\right|_{t}:=\Psi_{\uparrow U_{t}}$ is a biholomorphic map from $U_{t}$ to $B_{u(t)}\left(T_{\mathbb{R}}\right)$ and $\Psi(u(t))=0_{u(t)}$ holds.

(3)

$$
\sup _{t \in T, \dot{p} \in B_{u(t)}\left(T_{\mathbb{R}}\right)}\left\|\left(D\left(\left.\Psi\right|_{t} ^{-1}\right)\right)_{\dot{p}}\right\|_{L\left(\left(T_{\mathbb{R}}\right)_{u(t)}\left(\pi^{-1}(\{t\})\right) ;\left(T_{\mathbb{R}}\right)_{\left.\Psi\right|_{t} ^{\mid-1}(\dot{p})}\left(\pi^{-1}(\{t\})\right)\right)}<+\infty
$$

holds.

(4) For any $r \in[0,1)$ and $\varepsilon>0$, there exists $\delta>0$ such that for any $t \in T, p \in U_{t}$ and $q \in \pi^{-1}(\{t\})$,

$$
\begin{gathered}
\|\Psi(p)\|_{u(t)} \leq r, \quad d_{t}(q, p)<\delta \\
\Rightarrow \quad q \in U_{t}, \quad\|\Psi(q)-\Psi(p)\|_{u(t)}<\varepsilon
\end{gathered}
$$

holds.

Definition 3.4 (Coordinate neighborhood of the set of all continuous sections defined by a holomorphic normal coordinate neighborhood) :

Let $T$ be a non-empty subset of $X$. Let $u \in \Gamma(M \mid T)$. Let $(U, \Psi)$ to be a holomorphic normal coordinate neighborhood of $u$. Then, let

$$
\tilde{U}:=\left\{v \in \Gamma(M \mid T) \mid \exists r \in[0,1), \forall t \in T: v(t) \in U_{t},\|\Psi(v(t))\|_{u(t)} \leq r\right\} .
$$

A map $\tilde{\Psi}: \tilde{U} \rightarrow \Gamma_{b}\left(u^{\star}\left(T_{\mathbb{R}}(M)\right)\right)$ is defined as

$$
(\tilde{\Psi}(v))(t):=\Psi(v(t)) \quad(v \in \tilde{U}, t \in T) .
$$

$(\tilde{U}, \tilde{\Psi})$ is called the coordinate neighborhood of $\Gamma(M \mid T)$ defined by $(U, \Psi)$.

The next proposition is the technical main result of this paper. This proof is given in the next section.

Proposition 3.5 (Existence of a holomorphic normal coordinate neighborhood) :

Let $T$ be a non-empty normal subset of $X$. Let $u \in \Gamma(M \mid T)$. Then, there exists a holomorphic normal coordinate neighborhood of $u$.

Proof : It is given in the next section.

On the other hand, the main result of this paper is as follows.

Theorem 3.6 (Main result) :

Let $T$ be a non-empty normal subset of $X$. Then, the metric space $\Gamma(M \mid T)$ becomes a $C_{b}(T)$-manifold according to all coordinate neighborhoods of $\Gamma(M \mid T)$ defined by holomorphic normal coordinate neighborhoods.

For the proof of this theorem, Proposition 3.5 and the following lemma are used.

\section{Lemma 3.7 :}

Let $\varepsilon>0$. Let $X$ and $Y$ be $n$-dimensional complex inner product spaces. Let $f$ be a holomorphic map from $\left\{z \in X \mid\|z\|_{X}<\varepsilon\right\}$ to $\left\{w \in Y \mid\|w\|_{Y}<1\right\}$. Then,

$$
\left\|(D f)_{0}\right\|_{L(X ; Y)} \leq \frac{4 n^{2}}{\varepsilon}
$$


holds and

$$
\begin{gathered}
z \in X,\|z\|_{X} \leq \frac{1}{4} \varepsilon \\
\Rightarrow\left\|f(z)-\left(f(0)+(D f)_{0}(z)\right)\right\|_{Y} \leq \frac{16 n^{3}}{\varepsilon^{2}}\|z\|_{X}^{2}
\end{gathered}
$$

holds.

Proof : Taking an orthonormal basis of $X$ and $Y$, respectively, we consider that $X=Y=\mathbb{C}^{n}$ holds. $\|z\|_{X}=\|z\|_{\mathbb{C}^{n}}$ and $\|w\|_{Y}=\|w\|_{\mathbb{C}^{n}}$ holds. $f_{k}$ denotes the $k$-th component of $f .\left\{e_{k}\right\}_{k=1}^{n}$ denotes the canonical $\mathbb{Z}$-basis of $\mathbb{Z}^{n}$, that is, $\left\{e_{k}\right\}_{k=1}^{n}$ is the identity matrix of the order $n$.

Now, as $z \in \mathbb{C}^{n}$ and $\|z\|_{\mathbb{C}^{n}} \leq \frac{1}{2} \varepsilon$ hold, $\left|\frac{\partial f_{i}}{\partial z_{j}}(z)\right|=\left|\frac{2}{\pi \varepsilon} \int_{\theta \in[0,2 \pi]} e^{-\sqrt{-1} \theta} f_{i}\left(z+\frac{1}{4} \varepsilon e^{\sqrt{-1} \theta} e_{j}\right) d \theta\right| \leq \frac{4}{\varepsilon}$ holds. So,

$$
\|z\|_{\mathbb{C}^{n}} \leq \frac{1}{2} \varepsilon \Rightarrow\left|\frac{\partial f_{i}}{\partial z_{j}}(z)\right| \leq \frac{4}{\varepsilon}
$$

holds. Similarly, as $z \in \mathbb{C}^{n},\|z\|_{\mathbb{C}^{n}} \leq \frac{1}{4} \varepsilon$ and $s \in[0,1]$ hold, $\left|\frac{\partial^{2} f_{i}}{\partial z_{j} \partial z_{k}}(s z)\right|=\mid \frac{2}{\pi \varepsilon} \int_{\theta \in[0,2 \pi]} e^{-\sqrt{-1} \theta} \frac{\partial f_{i}}{\partial z_{k}}(s z+$ $\left.\frac{1}{4} \varepsilon e^{\sqrt{-1} \theta} e_{j}\right) d \theta \mid \leq \frac{16}{\varepsilon^{2}}$ and $\left|f_{i}(z)-\left(f_{i}(0)+\left(D f_{i}\right)_{0}(z)\right)\right|=\left|\sum_{j, k}\left(\int_{0}^{1}\left(\frac{\partial^{2} f_{i}}{\partial z_{j} \partial z_{k}}(s z)\right)(1-s) d s\right) z_{j} z_{k}\right| \leq \frac{16 n^{2}}{\varepsilon^{2}}\|z\|_{\mathbb{C}^{n}}^{2}$ hold. So,

$$
\|z\|_{\mathbb{C}^{n}} \leq \frac{1}{4} \varepsilon \Rightarrow\left|f_{i}(z)-\left(f_{i}(0)+\left(D f_{i}\right)_{0}(z)\right)\right| \leq \frac{16 n^{2}}{\varepsilon^{2}}\|z\|_{\mathbb{C}^{n}}^{2}
$$

holds.

Proof of Theorem 3.6 :

$1^{\circ}$ : Let $u \in \Gamma(M \mid T)$. Let

$$
\tilde{B}_{u}:=\left\{\dot{u} \in \Gamma_{b}\left(u^{*}\left(T_{\mathbb{R}}(M)\right)\right) \mid\|\dot{u}\|_{u}<1\right\} .
$$

Let $(U, \Psi)$ be a holomorphic normal coordinate neighborhood of $u$. Then, we show

$$
\tilde{\Psi}(\tilde{U}) \subset \tilde{B}_{u}
$$

Let $v \in \tilde{U} . v: T \rightarrow U$ and $\Psi: U \rightarrow u^{*}\left(T_{\mathbb{R}}(M)\right)$ are continuous. Also, there exists $r \in[0,1)$ such that $\sup _{t \in T}\|\Psi(v(t))\|_{u(t)} \leq r$ holds. Thus, $\tilde{\Psi}(v)=\Psi \circ v \in \tilde{B}_{u}$ holds.

$2^{\circ}$ : Let $u \in \Gamma(M \mid T)$. Let $(U, \Psi)$ be a holomorphic normal coordinate neighborhood of $u$. A map $\tilde{\Psi^{-1}}$ : $\tilde{B}_{u} \rightarrow \Gamma(M \mid T)$ is defined as

$$
\left(\tilde{\Psi^{-1}}(\dot{u})\right)(t):=\Psi^{-1}(\dot{u}(t)) \quad\left(\dot{u} \in \tilde{B}_{u}, t \in T\right) .
$$

Then, we show

$$
\tilde{\Psi^{-1}}\left(\tilde{B}_{u}\right) \subset \tilde{U} .
$$

Let $\dot{u} \in \tilde{B}_{u} \dot{u}: T \rightarrow \cup_{p \in u(T)} B_{p}\left(T_{\mathbb{R}}\right)$ and $\Psi^{-1}: \cup_{p \in u(T)} B_{p}\left(T_{\mathbb{R}}\right) \rightarrow U$ are continuous. Also, $\|\dot{u}\|_{u} \in[0,1)$ and $\|\dot{u}(t)\|_{u(t)} \leq\|\dot{u}\|_{u}$ hold. Thus, $\tilde{\Psi^{-1}}(\dot{u})=\Psi^{-1} \circ \dot{u} \in \tilde{U}$ holds.

$3^{\circ}$ : From $1^{\circ}$ and $2^{\circ}$,

$$
\tilde{\Psi^{-1}} \circ \tilde{\Psi}=1_{\tilde{U}}, \quad \tilde{\Psi} \circ \tilde{\Psi^{-1}}=1_{\tilde{B}_{u}}
$$

hold.

$4^{\circ}$ : We show that $\tilde{\Psi^{-1}}$ is Lipschitz continuous.

Let $\dot{u}_{0}, \dot{u}_{1} \in \tilde{B}_{u}$. Then, for any $s \in[0,1]$,

$$
\dot{u_{s}}:=\dot{u_{0}}+s\left(\dot{u_{1}}-\dot{u_{0}}\right)=(1-s) \dot{u_{0}}+s \dot{u_{1}} \in \tilde{B}_{u}
$$

holds. Thus,

$$
\begin{aligned}
& d_{t}\left(\left(\tilde{\Psi^{-1}}\left(\dot{u_{0}}\right)\right)(t),\left(\tilde{\Psi^{-1}}\left(\dot{u_{1}}\right)\right)(t)\right) \\
= & d_{t}\left(\Psi^{-1}\left(\dot{u_{0}}(t)\right), \Psi^{-1}\left(\dot{u_{1}}(t)\right)\right) \\
\leq & \int_{0}^{1}\left\|\frac{d}{d s}\left(\Psi^{-1}\left(\dot{u}_{s}(t)\right)\right)\right\|_{\Psi^{-1}\left(\dot{u}_{s}(t)\right)} d s
\end{aligned}
$$




$$
\begin{aligned}
& =\int_{0}^{1}\left\|\left(D\left(\left.\Psi\right|_{t} ^{-1}\right)\right)_{\dot{u}_{s}(t)}\left(\dot{u}_{1}(t)-\dot{u}_{0}(t)\right)\right\|_{\left.\Psi\right|_{t} ^{-1}\left(\dot{u}_{s}(t)\right)} d s \\
& \leq\left(\int_{0}^{1}\left\|\left(D\left(\left.\Psi\right|_{t} ^{-1}\right)\right)_{\dot{u}_{s}(t)}\right\|_{L\left(\left(T_{\mathbb{R}}\right)_{u(t)}\left(\pi^{-1}(\{t\})\right) ;\left(T_{\mathbb{R}}\right)_{\left.\Psi\right|_{t} ^{-1}\left(\dot{u}_{s}(t)\right)}\left(\pi^{-1}(\{t\})\right)\right)} d s\right)\left\|\dot{u}_{1}(t)-\dot{u}_{0}(t)\right\|_{u(t)}
\end{aligned}
$$

holds. Therefore,

$$
\begin{gathered}
d_{T}\left(\tilde{\Psi^{-1}}\left(\dot{u_{0}}\right), \tilde{\Psi^{-1}}\left(\dot{u_{1}}\right)\right) \\
\left.\leq \sup _{t \in T, \dot{p} \in B_{u(t)}\left(T_{\mathbb{R}}\right)}\left\|\left(D\left(\left.\Psi\right|_{t} ^{-1}\right)\right)_{\dot{p}}\right\|_{L\left(\left(T_{\mathbb{R}}\right)_{u(t)}\left(\pi^{-1}(\{t\})\right) ;\left(T_{\mathbb{R}}\right)_{\left.\Psi\right|_{t} ^{\mid}}^{-1(\dot{p})}\left(\pi^{-1}(\{t\})\right)\right)}\right)\left\|\dot{u_{1}}-\dot{u_{0}}\right\|_{u}
\end{gathered}
$$

holds.

$5^{\circ}$ : We show that $\tilde{\Psi}$ is continuous.

Let $v \in \tilde{U}$ and $\varepsilon>0$. Then, there exists $r \in[0,1)$ such that for any $t \in T$,

$$
v(t) \in U_{t}, \quad\|\Psi(v(t))\|_{u(t)} \leq r
$$

hold. Therefore, there exists $\delta>0$ such that for any $t \in T$ and $q \in \pi^{-1}(\{t\})$,

$$
\begin{gathered}
d_{t}(q, v(t))<\delta \\
\Rightarrow \quad q \in U_{t}, \quad\|\Psi(q)-\Psi(v(t))\|_{u(t)}<\frac{\varepsilon}{2}
\end{gathered}
$$

holds. Let $w \in \tilde{U}$ and $d_{T}(w, v)<\delta$. Then, because of $d_{t}(w(t), v(t))<\delta$,

$$
\|\Psi(w(t))-\Psi(v(t))\|_{u(t)}<\frac{\varepsilon}{2}
$$

holds. So,

$$
\|\tilde{\Psi}(w)-\tilde{\Psi}(v)\|_{u} \leq \frac{\varepsilon}{2}<\varepsilon
$$

holds.

$6^{\circ}$ : We show that $\tilde{U}$ is an open set of $\Gamma(M \mid T)$.

Let $v \in \tilde{U}$. Then, there exists $r \in[0,1)$ such that for any $t \in T$,

$$
v(t) \in U_{t}, \quad\|\Psi(v(t))\|_{u(t)} \leq r
$$

hold. Thus, there exists $\varepsilon>0$ such that for any $t \in T$ and $q \in \pi^{-1}(\{t\})$,

$$
\begin{gathered}
d_{t}(q, v(t))<\varepsilon \\
\Rightarrow \quad q \in U_{t}, \quad\|\Psi(q)-\Psi(v(t))\|_{u(t)}<\frac{1-r}{2}
\end{gathered}
$$

holds. Let $w \in \Gamma(M \mid T)$ and $d_{T}(w, v)<\varepsilon$. Then, because of $d_{t}(w(t), v(t))<\varepsilon$,

$$
w(t) \in U_{t}, \quad\|\Psi(w(t))-\Psi(v(t))\|_{u(t)}<\frac{1-r}{2}
$$

hold. Therefore, furthermore,

$$
\begin{gathered}
\|\Psi(w(t))\|_{u(t)} \\
\leq\|\Psi(w(t))-\Psi(v(t))\|_{u(t)}+\|\Psi(v(t))\|_{u(t)} \\
<\frac{1-r}{2}+r=\frac{1+r}{2}
\end{gathered}
$$

holds, while $\frac{1+r}{2} \in[0,1)$ holds. Hence, $w \in \tilde{U}$ holds.

$7^{\circ}: \quad \tilde{B}_{u}$ is an open set of $\Gamma_{b}\left(u^{\star}\left(T_{\mathbb{R}}(M)\right)\right)$. On the other hand, from $\Psi(u(t))=0_{u(t)}$, we obtain $u \in \tilde{U}$. Therefore, by Proposition 3.5, $\Gamma(M \mid T)$ is a topological manifold according to all coordinate neighborhoods defined by holomorphic normal coordinate neighborhoods. 
$8^{\circ}$ : We show that each coordinate transformation is $C_{b}(T)$-differentiable (i.e., it is Fréchet differentiable and its Fréchet derivatives are $C_{b}(T)$-linear).

Let $(U, \Psi)$ be a holomorphic normal coordinate neighborhood of $u$. Let $(V, \Phi)$ be a holomorphic normal coordinate neighborhood of $v$. Let $\dot{u} \in \tilde{\Psi}(\tilde{U} \cap \tilde{V})$. Then, there exists $\varepsilon>0$ such that for any $\dot{h} \in \Gamma_{b}\left(u^{\star}\left(T_{\mathbb{R}}(M)\right)\right)$,

$$
\|\dot{h}\|_{u}<\varepsilon \Rightarrow \dot{u}+\dot{h} \in \tilde{\Psi}(\tilde{U} \cap \tilde{V})
$$

holds. Also, because $T$ is normal, if $\|\dot{p}\|_{u(t)}<\varepsilon$ holds, then there exists $\dot{h} \in \Gamma_{b}\left(u^{\star}\left(T_{\mathbb{R}}(M)\right)\right)$ such that

$$
\|\dot{h}\|_{u}<\varepsilon, \quad \dot{h}(t)=\dot{p}
$$

hold. (For this, since we can take a local trivialization coordinate system of the vector bundle $u^{\star}\left(T_{\mathbb{R}}(M)\right.$ ) and make the Schmidt orthogonalization on the canonical basis at each $t$ to obtain an isomorphism to the product bundle of the complex inner product space, it is only necessary to paste the constant section through $\dot{p}$ and the zero section by a partition of unity.) Hence,

$$
\|\dot{p}\|_{u(t)}<\varepsilon \Rightarrow \dot{u}(t)+\left.\dot{p} \in \Psi\right|_{t}\left(U_{t} \cap V_{t}\right)
$$

holds. So, by Lemma 3.7,

$$
\left\|\left(D\left(\left.\left.\Phi\right|_{t} \circ \Psi\right|_{t} ^{-1}\right)\right)_{\dot{u}(t)}\right\|_{L\left(\left(T_{\mathbb{R}}\right)_{u(t)}\left(\pi^{-1}(\{t\})\right) ;\left(T_{\mathbb{R}}\right)_{v(t)}\left(\pi^{-1}(\{t\})\right)\right)} \leq \frac{4 n^{2}}{\varepsilon}
$$

holds and also,

$$
\begin{gathered}
\|\dot{p}\|_{u(t)} \leq \frac{1}{4} \varepsilon \\
\Rightarrow \quad \\
\left\|\left(\left.\left.\Phi\right|_{t} \circ \Psi\right|_{t} ^{-1}\right)(\dot{u}(t)+\dot{p})-\left(\left(\left.\left.\Phi\right|_{t} \circ \Psi\right|_{t} ^{-1}\right)(\dot{u}(t))+\left(D\left(\left.\left.\Phi\right|_{t} \circ \Psi\right|_{t} ^{-1}\right)\right)_{\dot{u}(t)}(\dot{p})\right)\right\|_{v(t)} \\
\leq \frac{16 n^{3}}{\varepsilon^{2}}\|\dot{p}\|_{u(t)}^{2}
\end{gathered}
$$

holds.

Now, for $\dot{h} \in \Gamma_{b}\left(u^{\star}\left(T_{\mathbb{R}}(M)\right)\right)$ and $t \in T$, we define

$$
(\tilde{A}(\dot{h}))(t):=\left(D\left(\left.\left.\Phi\right|_{t} \circ \Psi\right|_{t} ^{-1}\right)\right)_{\dot{u}(t)}(\dot{h}(t)) .
$$

Then, $(\tilde{A}(\dot{h}))(t) \in\left(T_{\mathbb{R}}\right)_{v(t)}\left(\pi^{-1}(\{t\})\right)$ holds. Furthermore, in virtue of Lemma 1.11, the section

$$
t \in T \mapsto(\tilde{A}(\dot{h}))(t) \in v^{*}\left(T_{\mathbb{R}}(M)\right)
$$

is continuous. (This is because taking a respective local trivialization coordinate system of the vector bundle $u^{\star}\left(T_{\mathbb{R}}(M)\right)$ and $v^{\star}\left(T_{\mathbb{R}}(M)\right)$ and displaying the map $\dot{p} \mapsto\left(\Phi \circ \Psi^{-1}\right)(\dot{p})$ in the local coordinates to be holomorphic on each fiber and to be continuous, so that the map $\dot{p} \mapsto\left(D\left(\left.\left.\Phi\right|_{\pi(\varpi(\dot{p}))} \circ \Psi\right|_{\pi(\varpi(\dot{p}))} ^{-1}\right)\right)_{\dot{p}}$ is also continuous.) Also, while it is obvious that $\tilde{A}$ is $C_{b}(T)$-linear, from (3.1), $\tilde{A}$ is a continuous map from $\Gamma_{b}\left(u^{\star}\left(T_{\mathbb{R}}(M)\right)\right)$ to $\Gamma_{b}\left(v^{\star}\left(T_{\mathbb{R}}(M)\right)\right)$. However, from (3.2), $\tilde{A}$ is the Fréchet derivative of $\tilde{\Phi} \circ \tilde{\Psi^{-1}}$ at the point $\dot{u}$.

Corollary 3.8 :

Suppose that $T$ is a paracompact contractible subset of $X$. Then, $\Gamma(M \mid T)$ is an $n$-dimensional $C_{b}(T)$ manifold.

Proof : Let $u \in \Gamma(M \mid T)$. Then, as a continuous complex vector bundle on $T, u^{*}\left(T_{\mathbb{R}}(M)\right)$ is isomorphic to $\mathbb{C}^{n} \times T$. That is, there exists $\left\{\dot{u}_{k}\right\}_{k=1}^{n}$ such that $\dot{u}_{k} \in \Gamma\left(u^{*}\left(T_{\mathbb{R}}(M)\right)\right)$ holds and for any $t \in T,\left\{\dot{u}_{k}(t)\right\}_{k=1}^{n}$ is a basis of $\left(T_{\mathbb{R}}\right)_{u(t)}\left(\pi^{-1}(\{t\})\right)$. We denote the Schmidt orthogonalization on $\left\{\dot{u}_{k}(t)\right\}_{k=1}^{n}$ at each $t \in T$ by $\left\{\dot{e}_{k}(t)\right\}_{k=1}^{n}$. Then, $\dot{e}_{k} \in \Gamma_{b}\left(u^{*}\left(T_{\mathbb{R}}(M)\right)\right)$ holds and for any $t \in T,\left\{\dot{e}_{k}(t)\right\}_{k=1}^{n}$ is an orthonormal basis of $\left(T_{\mathbb{R}}\right)_{u(t)}\left(\pi^{-1}(\{t\})\right)$. We define a map $F:\left(C_{b}(T)\right)^{n} \rightarrow \Gamma_{b}\left(u^{*}\left(T_{\mathbb{R}}(M)\right)\right)$ as

$$
F\left(\left\{\dot{z}_{1}(t)\right\}_{t \in T},\left\{\dot{z}_{2}(t)\right\}_{t \in T}, \cdots,\left\{\dot{z}_{n}(t)\right\}_{t \in T}\right)
$$




$$
:=\left\{\sum_{k} \dot{z}_{k}(t) \dot{e}_{k}(t)\right\}_{t \in T}
$$

Because of $\max _{k}\left|z_{k}\right| \leq\|z\|_{\mathbb{C}^{n}} \leq n \max _{k}\left|z_{k}\right|$, as in Example 2.9, $F$ and $F^{-1}$ are continuous $C_{b}(T)$-linear maps.

Remark (Serre-Swan theorem) :

By making a continuous complex vector bundle $M$ on $X$ correspond to the module $\Gamma(M)$ of all continuous sections of $M$ on $X$, the category of continuous complex vector bundles on $X$ is equivalent to the one of finitely generated projective $C(X)$-modules $([2,11,13,14])$.

Remark (Manifold on the real commutative algebra $\left.C^{\infty}(X ; \mathbb{R})\right)$ :

Let $M \rightarrow X$ be a differentiable family of differentiable manifolds. The set of all differentiable sections of $M$ on $X$ becomes a $C^{\infty}(X ; \mathbb{R})$-manifold $([4,8,9,10])$. However, $C^{\infty}(X ; \mathbb{R})$ is not a real Banach space, but it is a real Fréchet space.

Remark : We did a little study on $A$-manifolds and $\mathbb{C}^{n}$-holomorphic functions $([15,16])$.

\section{Corollary 3.9 :}

Suppose $X \neq \emptyset$. Then, the set of all continuous sections of $M$ on $X$ is a $C(X)$-manifold.

Proof : It follows from Theorem 3.6.

Corollary 3.10 :

Suppose that $X$ is contractible. Then, the set of all continuous sections of $M$ on $X$ is an $n$-dimensional $C(X)$-manifold.

Proof : It follows from Corollary 3.8.

\section{Holomorphic linear connections}

In this section, we prove Proposition 3.5. That is, we have to show the existence of a holomorphic normal coordinate neighborhood. For that, we use sprays. However, the global spray corresponding to Levi-Civita connection may not be holomorphic. For each section, we construct a holomorphic spray on its neighborhood as the one corresponding to a convex combination of trivial connections.

Definition 4.1 (Holomorphic linear spray) :

Let $Z$ be a real $C^{\infty}$-vector field on the real tangent bundle $T_{\mathbb{R}}(N)$ of an $n$-dimensional complex manifold $N$. Then, $Z$ is said to be a holomorphic linear spray on $N$, if for any holomorphic local coordinate system $z=\left(z_{1}, z_{2}, \cdots, z_{n}\right)$ of $N$, there exists a family $\left\{\Gamma_{i, j}^{k}\right\}_{i, j, k \in\{1,2, \cdots, n\}}$ of holomorphic functions on the coordinate neighborhood of $z$ such that

$$
\Gamma_{i, j}^{k}=\Gamma_{j, i}^{k} \quad(i, j, k=1,2, \cdots, n)
$$

holds and any integral curve $\{(\dot{z}(s), z(s))\}_{s \in(-\varepsilon,+\varepsilon)}$ of the vector field $Z$ satisfies the ordinal differential equation

$$
\begin{gathered}
\frac{d}{d s} \dot{z}_{k}=-\sum_{i, j} \Gamma_{i, j}^{k}\left(z_{1}, z_{2}, \cdots, z_{n}\right) \dot{z}_{i} \dot{z}_{j}, \\
\frac{d}{d s} z_{k}=\dot{z}_{k} .
\end{gathered}
$$

Again, recall that a continuous family $M\left(=\left(M, X, \pi, S,\left\{\left(\rho_{\varphi}, M_{\varphi}^{\prime}, X_{\varphi}^{\prime}\right)\right\}_{\varphi \in S}, r_{0}\right)\right)$ with bumps was fixed.

Definition 4.2 (Trivial spray) :

(1) Let $\varphi \in S$. Let

$$
\begin{gathered}
N_{\varphi}:=\left(\varphi,\left.\pi\right|_{\varphi}\right)^{-1}\left(D_{\frac{r_{0}+1}{2}}^{n} \times \overline{X_{\varphi}^{\prime}}\right) \\
=\varphi^{-1}\left(D_{\frac{r_{0}+1}{2}}^{n}\right) \cap \pi^{-1}\left(\overline{X_{\varphi}^{\prime}}\right) .
\end{gathered}
$$

$N_{\varphi}$ is an open set of $\pi^{-1}\left(\overline{X_{\varphi}^{\prime}}\right)$. Further, let $t \in X$. Let

$$
N_{\varphi, t}:=N_{\varphi} \cap \pi^{-1}(\{t\})
$$




$$
=\left\{\begin{array}{cr}
\left.\varphi\right|_{t} ^{-1}\left(D_{\frac{r_{0}+1}{2}}^{n}\right) & \left(t \in \overline{X_{\varphi}^{\prime}}\right), \\
\emptyset & \left(t \in X \backslash \overline{X_{\varphi}^{\prime}}\right) .
\end{array}\right.
$$

$N_{\varphi, t}$ is an open set of the $n$-dimensional complex manifold $\pi^{-1}(\{t\})$.

(2) Let $\varphi \in S$ and $t \in X$. A real $C^{\infty}$-vector field $Y_{\varphi, t}$ on the real tangent bundle $T_{\mathbb{R}}\left(N_{\varphi, t}\right)$ is determined to satisfy the following. With respect to the holomorphic coordinate system $p \in N_{\varphi, t} \mapsto z=\varphi(p) \in \mathbb{C}^{n}$ of $N_{\varphi, t}$, any integral curve $(\dot{z}, z)$ of $Y_{\varphi, t}$ satisfies the ordinary differential equation

$$
\frac{d}{d s} \dot{z}=0, \quad \frac{d}{d s} z=\dot{z} .
$$

\section{Proposition 4.3 :}

(1) The trivial spray $Y_{\varphi, t}$ is a holomorphic linear one on $N_{\varphi, t}$.

(2) Let $\psi \in S$. With respect to the holomorphic local coordinate system $p \in N_{\psi, t} \cap N_{\varphi, t} \mapsto z=\psi(p) \in$ $\mathbb{C}^{n}$ of $N_{\varphi, t}$, any integral curve $(\dot{z}, z)$ of the trivial spray $Y_{\varphi, t}$ satisfies the ordinary differential equation

$$
\begin{gathered}
\frac{d}{d s} \dot{z}_{k}=-\sum_{i, j}(\Gamma(\varphi, \psi))_{i, j}^{k}(z, t) \dot{z}_{i} \dot{z}_{j}, \\
\frac{d}{d s} z_{k}=\dot{z}_{k},
\end{gathered}
$$

provided that $\left.\varphi\right|_{t_{(i)}}$ is the $i$-th component of $\left.\varphi\right|_{t},\left.\psi\right|_{t_{(j)}}$ is the $j$-th component of $\left.\psi\right|_{t}$ and

$$
\begin{gathered}
(\Gamma(\varphi, \psi))_{i, j}^{k}(z, t) \\
:=-\sum_{l, m}\left(\left(\partial_{z_{l}} \partial_{z_{m}}\left(\left.\left.\psi\right|_{t_{(k)}} \circ \varphi\right|_{t} ^{-1}\right)\right)\left(\left(\left.\left.\varphi\right|_{t} \circ \psi\right|_{t} ^{-1}\right)(z)\right)\right) \\
\left(\left(\partial_{z_{i}}\left(\left.\left.\varphi\right|_{t_{(l)}} \circ \psi\right|_{t} ^{-1}\right)\right)(z)\right)\left(\left(\partial_{z_{j}}\left(\left.\left.\varphi\right|_{t_{(m)}} \circ \psi\right|_{t} ^{-1}\right)\right)(z)\right)
\end{gathered}
$$

holds.

Proof : Simple calculation.

Lemma 4.4 :

Let $(\Gamma(\varphi, \psi))_{i, j}^{k}$ be the same as Proposition 4.3. Then, the function

$$
(z, t) \in\left(\psi,\left.\pi\right|_{\psi}\right)\left(N_{\varphi} \cap N_{\psi}\right) \mapsto(\Gamma(\varphi, \psi))_{i, j}^{k}(z, t) \in \mathbb{C}
$$

is continuous.

Proof: According to Lemma 1.11.

Definition 4.5 (Weighting space) :

Let $R$ be a non-empty subset of $S$. Let

$$
W_{R}:=\left\{c \in[0,1]^{S} \mid \sum_{\varphi \in S} c_{\varphi}=\sum_{\varphi \in R} c_{\varphi}=1\right\} .
$$

$W_{R}$ is called the weighting space of $R$ and an element of $W_{R}$ is called a weighting of $R$.

Remark : $W_{R}$ is a compact subset of $[0,1]^{S} . R_{1} \subset R_{2}$ implies $W_{R_{1}} \subset W_{R_{2}}$.

Definition 4.6 (Spray determined by a weighting) :

(1) Let $R$ be a non-empty subset of $S$. Let

$$
N^{R}:=\cap_{\varphi \in R} N_{\varphi}=\left(\cap_{\varphi \in R} \varphi^{-1}\left(D_{\frac{r_{0}+1}{2}}^{n}\right)\right) \cap \pi^{-1}\left(\cap_{\varphi \in R} \overline{X_{\varphi}^{\prime}}\right) .
$$

$N^{R}$ is an open set of $\pi^{-1}\left(\cap_{\varphi \in R} \overline{X_{\varphi}^{\prime}}\right)$. Further, let $t \in X$. Let

$$
N_{t}^{R}:=\cap_{\varphi \in R} N_{\varphi, t}=N^{R} \cap \pi^{-1}(\{t\})
$$




$$
=\left\{\begin{array}{cr}
\cap_{\varphi \in R}\left(\left.\varphi\right|_{t} ^{-1}\left(D_{\frac{r_{0}+1}{2}}^{n}\right)\right) & \left(t \in \cap_{\varphi \in R} \overline{X_{\varphi}^{\prime}}\right), \\
\emptyset & \left(t \in X \backslash \cap_{\varphi \in R} \overline{X_{\varphi}^{\prime}}\right) .
\end{array}\right.
$$

$N_{t}^{R}$ is an open set of the $n$-dimensional complex manifold $\pi^{-1}(\{t\})$.

(2) For $c \in W_{S}$ and $t \in X$, a holomorphic linear spray $Y^{c, t}$ on $N_{t}^{c^{-1}((0,1])}$ is defined as

$$
Y^{c, t}:=\sum_{\varphi \in S} c_{\varphi} Y_{\varphi, t} .
$$

For a non-empty subset $R$ of $S, c \in W_{R}$ and $t \in X$, a holomorphic linear spray $Y_{R}^{c, t}$ on $N_{t}^{R}$ is defined as

$$
Y_{R}^{c, t}:=Y^{c, t}{ }_{\left\lceil N_{t}^{R}\right.}=\sum_{\varphi \in R} c_{\varphi} Y_{\varphi, t_{\left\lceil N_{t}^{R}\right.}} .
$$

Remark : When $c \in W_{R}$ holds, $c^{-1}((0,1]) \subset R$ and $N_{t}^{R} \subset N_{t}^{c^{-1}((0,1])}$ hold.

Definition 4.7 (Coordinate display of the spray) :

Let $\psi \in R \subset S$. Let

$$
\begin{gathered}
N^{R, \psi}:=\left(\psi,\left.\pi\right|_{\psi}\right)\left(N^{R}\right), \\
U^{R, \psi}:=\mathbb{C}^{n} \times N^{R, \psi} \times W_{R} .
\end{gathered}
$$

$N^{R, \psi}$ is an open set of $D_{\frac{r_{0}+1}{2}}^{n} \times\left(\cap_{\varphi \in R} \overline{X_{\varphi}^{\prime}}\right)$ and $U^{R, \psi}$ is an open set of $\left(\mathbb{C}^{n} \times D_{\frac{r_{0+1}}{2}}^{n}\right) \times\left(\left(\cap_{\varphi \in R} \overline{X_{\varphi}^{\prime}}\right) \times W_{R}\right)$. Let $\left\{(\Gamma(\varphi, \psi))_{i, j}^{k}\right\}_{\varphi, \psi, i, j, k}$ be the same as Proposition 4.3. A map

$$
f^{R, \psi}: U^{R, \psi} \rightarrow \mathbb{C}^{2 n}
$$

is defined as

$$
\begin{gathered}
f_{k}^{R, \psi}(\dot{z}, z, t, c):=-\sum_{i, j}\left(\sum_{\varphi \in R} c_{\varphi}(\Gamma(\varphi, \psi))_{i, j}^{k}(z, t)\right) \dot{z}_{i} \dot{z}_{j} \quad(k=1,2, \cdots, n), \\
f_{k}^{R, \psi}(\dot{z}, z, t, c):=\dot{z}_{k-n} \quad(k=n+1, n+2, \cdots, 2 n) .
\end{gathered}
$$

Definition 4.8 (Coordinate display of the exponential map) :

Let $\psi \in R \subset S$. Then, the set of all $(\dot{z}, z, t, c) \in U^{R, \psi}\left(=\mathbb{C}^{n} \times N^{R, \psi} \times W_{R}\right)$ such that there exists $(\dot{w}, w)$ : $[0,1] \rightarrow \mathbb{C}^{n} \times D_{\frac{r_{0+1}}{2}}^{n}$ such that

$$
\frac{d}{d s}(\dot{w}, w)=f^{R, \psi}(\dot{w}, w, t, c) \quad(s \in[0,1]),
$$

$$
(\dot{w}, w)(0)=(\dot{z}, z)
$$

hold is denoted by $I^{R, \psi}$. Also, a map

$$
\left(\dot{e}^{R, \psi}, e^{R, \psi}\right): I^{R, \psi} \rightarrow \mathbb{C}^{n} \times D_{\frac{r_{0}+1}{2}}^{n}
$$

is defined by $\left(\dot{e}^{R, \psi}, e^{R, \psi}\right)(\dot{z}, z, t, c)=(\dot{w}, w)(1)$.

Lemma 4.9 (ODE with a continuous parameter) :

Let $\Lambda$ be a topological space. Let $U$ be an open set of $\mathbb{R}^{m} \times \Lambda$. Suppose that $f: U \rightarrow \mathbb{R}^{m}$ is a continuous map. Suppose that for any $\lambda \in \Lambda$, the map $x \mapsto f(x, \lambda)$ is differentiable. Suppose that the map $D_{x} f: U \rightarrow$ $L\left(\mathbb{R}^{m} ; \mathbb{R}^{m}\right)$ is continuous. Further, let $\left(x_{0}, \lambda_{0}\right) \in U$. Suppose that $u_{0}:[0,1] \rightarrow \mathbb{R}^{m}$ is the solution of the initial value problem

$$
\frac{d}{d s} u=f\left(u, \lambda_{0}\right), \quad u(0)=x_{0} .
$$

Then, for any $\varepsilon>0$, there exists an open set $V$ of $U$ such that it satisfies the followings.

(1) $\left(x_{0}, \lambda_{0}\right) \in V$ holds. 
(2) For any $(x, \lambda) \in V$, there exists the solution $u:[0,1] \rightarrow \mathbb{R}^{m}$ of the initial value problem

$$
\frac{d}{d s} u=f(u, \lambda), \quad u(0)=x
$$

such that

$$
\sup _{s \in[0,1]}\left\|u(s)-u_{0}(s)\right\|_{\mathbb{R}^{m}}<\varepsilon
$$

holds.

Proof : Proofs of this type of lemma are often done by showing that the solutions can be extended using Gronwall inequalities, but here we see that the principle of contraction mappings works directly on an appropriate integral equation.

For any $s \in[0,1],\left(u_{0}(s), \lambda_{0}\right) \in U$ holds. The map

$$
s \in[0,1] \mapsto\left(D_{x} f\right)\left(u_{0}(s), \lambda_{0}\right) \in L\left(\mathbb{R}^{m} ; \mathbb{R}^{m}\right)
$$

is continuous. Hence, there exists $A:[0,1] \rightarrow L\left(\mathbb{R}^{m} ; \mathbb{R}^{m}\right)$ such that

$$
\frac{d}{d s} A(s)=\left(\left(D_{x} f\right)\left(u_{0}(s), \lambda_{0}\right)\right) A(s), \quad A(0)=1_{\mathbb{R}^{m}}
$$

hold. Further, $\operatorname{det}(A(s)) \neq 0$ and

$$
\begin{gathered}
1 \leq C_{1}:=\sup _{s \in[0,1]}\left\|(A(s))^{-1}\right\|_{L\left(\mathbb{R}^{m} ; \mathbb{R}^{m}\right)}<+\infty, \\
1 \leq C_{2}:=\sup _{s \in[0,1]}\|A(s)\|_{L\left(\mathbb{R}^{m} ; \mathbb{R}^{m}\right)}<+\infty
\end{gathered}
$$

hold. Then, for $s \in[0,1]$, there exist $\delta_{s}>0$ and an open set $\Lambda_{s}$ of $\Lambda$ such that $\lambda_{0} \in \Lambda_{s}$ and

$$
\begin{gathered}
s^{\prime} \in[0,1],\left|s^{\prime}-s\right|<\delta_{s}, x \in \mathbb{R}^{m},\|x\|_{\mathbb{R}^{m}}<2 \delta_{s}, \lambda \in \Lambda_{s} \\
\Rightarrow \\
\left\{\begin{array}{c}
\left(u_{0}\left(s^{\prime}\right)+A\left(s^{\prime}\right) x, \lambda\right) \in U, \\
\left\|\left(D_{x} f\right)\left(u_{0}\left(s^{\prime}\right)+A\left(s^{\prime}\right) x, \lambda\right)-\left(D_{x} f\right)\left(u_{0}(s), \lambda_{0}\right)\right\|_{L\left(\mathbb{R}^{m} ; \mathbb{R}^{m}\right)} \leq \frac{1}{4 C_{1} C_{2}}
\end{array}\right.
\end{gathered}
$$

hold. There exists a finite subset $L(\neq \emptyset)$ of $[0,1]$ such that

$$
s \in[0,1] \Rightarrow \exists s^{\prime} \in L:\left|s-s^{\prime}\right|<\delta_{s^{\prime}}
$$

holds. Now, we set

$$
\begin{aligned}
\delta^{\prime} & :=\min _{s \in L} \delta_{s}, \\
\Lambda^{\prime} & :=\cap_{s \in L} \Lambda_{s} .
\end{aligned}
$$

$\delta^{\prime}>0$ and $\lambda_{0} \in \Lambda^{\prime}$ hold. $\Lambda^{\prime}$ is an open set of $\Lambda$. Now, as $s \in[0,1], x \in \mathbb{R}^{m},\|x\|_{\mathbb{R}^{m}} \leq \delta^{\prime}$ and $\lambda \in \Lambda^{\prime}$ hold, because there exists $s^{\prime} \in L$ such that

$$
\left|s-s^{\prime}\right|<\delta_{s^{\prime}}
$$

holds, from $\lambda \in \Lambda^{\prime} \subset \Lambda_{s^{\prime}}$ and $\|x\|_{\mathbb{R}^{m}} \leq \delta^{\prime} \leq \delta_{s^{\prime}}<2 \delta_{s^{\prime}}$,

$$
\begin{gathered}
C_{1} C_{2}\left\|\left(D_{x} f\right)\left(u_{0}(s)+A(s) x, \lambda\right)-\left(D_{x} f\right)\left(u_{0}(s), \lambda_{0}\right)\right\|_{L\left(\mathbb{R}^{m} ; \mathbb{R}^{m}\right)} \\
\leq C_{1} C_{2}\left\|\left(D_{x} f\right)\left(u_{0}(s)+A(s) x, \lambda\right)-\left(D_{x} f\right)\left(u_{0}\left(s^{\prime}\right), \lambda_{0}\right)\right\|_{L\left(\mathbb{R}^{m} ; \mathbb{R}^{m}\right)} \\
+C_{1} C_{2}\left\|\left(D_{x} f\right)\left(u_{0}(s), \lambda_{0}\right)-\left(D_{x} f\right)\left(u_{0}\left(s^{\prime}\right), \lambda_{0}\right)\right\|_{L\left(\mathbb{R}^{m} ; \mathbb{R}^{m}\right)} \\
\leq \frac{1}{4}+\frac{1}{4} \leq \frac{1}{2}
\end{gathered}
$$


holds. That is,

$$
\begin{gathered}
s \in[0,1], x \in \mathbb{R}^{m},\|x\|_{\mathbb{R}^{m}} \leq \delta^{\prime}, \lambda \in \Lambda^{\prime} \\
\Rightarrow \\
\left\{\begin{array}{c}
\left(u_{0}(s)+A(s) x, \lambda\right) \in U, \\
C_{1} C_{2}\left\|\left(D_{x} f\right)\left(u_{0}(s)+A(s) x, \lambda\right)-\left(D_{x} f\right)\left(u_{0}(s), \lambda_{0}\right)\right\|_{L\left(\mathbb{R}^{m} ; \mathbb{R}^{m}\right)} \leq \frac{1}{2}
\end{array}\right.
\end{gathered}
$$

holds.

Now, let $\varepsilon>0$. Then, for $s \in[0,1]$, there exist $\delta_{s}^{\prime \prime}>0$ and an open set $\Lambda_{s}^{\prime \prime}$ of $\Lambda$ such that $\lambda_{0} \in \Lambda_{s}^{\prime \prime}$ and

$$
\begin{gathered}
s^{\prime \prime} \in[0,1],\left|s^{\prime \prime}-s\right|<\delta_{s}^{\prime \prime}, \lambda \in \Lambda_{s}^{\prime \prime} \\
\Rightarrow \\
\left\{\begin{array}{c}
\left(u_{0}\left(s^{\prime \prime}\right), \lambda\right) \in U, \\
\left\|f\left(u_{0}\left(s^{\prime \prime}\right), \lambda\right)-f\left(u_{0}(s), \lambda_{0}\right)\right\|_{\mathbb{R}^{m}} \leq \frac{1}{8 C_{1}} \min \left\{\delta^{\prime}, \frac{\varepsilon}{2 C_{2}}\right\}
\end{array}\right.
\end{gathered}
$$

hold. There exists a finite subset $L^{\prime \prime}(\neq \emptyset)$ of $[0,1]$ such that

$$
s \in[0,1] \Rightarrow \exists s^{\prime \prime} \in L^{\prime \prime}:\left|s-s^{\prime \prime}\right|<\delta_{s^{\prime \prime}}^{\prime \prime}
$$

holds. Now, we set

$$
\Lambda^{\prime \prime \prime}:=\cap_{s \in L^{\prime \prime}} \Lambda_{s}^{\prime \prime}
$$

$\lambda_{0} \in \Lambda^{\prime \prime \prime}$ holds. $\Lambda^{\prime \prime \prime}$ is an open set of $\Lambda$. Now, as $s \in[0,1]$ and $\lambda \in \Lambda^{\prime \prime \prime}$ hold, because there exists $s^{\prime \prime} \in L^{\prime \prime}$ such that

$$
\left|s-s^{\prime \prime}\right|<\delta_{s^{\prime \prime}}^{\prime \prime}
$$

holds, from $\lambda \in \Lambda^{\prime \prime \prime} \subset \Lambda_{s^{\prime \prime}}^{\prime \prime}$,

$$
\begin{aligned}
& C_{1}\left\|f\left(u_{0}(s), \lambda\right)-f\left(u_{0}(s), \lambda_{0}\right)\right\|_{\mathbb{R}^{m}} \\
& \leq C_{1}\left\|f\left(u_{0}(s), \lambda\right)-f\left(u_{0}\left(s^{\prime \prime}\right), \lambda_{0}\right)\right\|_{\mathbb{R}^{m}} \\
&+ C_{1}\left\|f\left(u_{0}(s), \lambda_{0}\right)-f\left(u_{0}\left(s^{\prime \prime}\right), \lambda_{0}\right)\right\|_{\mathbb{R}^{m}} \\
& \leq \frac{1}{8} \min \left\{\delta^{\prime}, \frac{\varepsilon}{2 C_{2}}\right\}+\frac{1}{8} \min \left\{\delta^{\prime}, \frac{\varepsilon}{2 C_{2}}\right\}=\frac{1}{4} \min \left\{\delta^{\prime}, \frac{\varepsilon}{2 C_{2}}\right\}
\end{aligned}
$$

holds. That is,

$$
\begin{gathered}
s \in[0,1], \lambda \in \Lambda^{\prime \prime \prime} \\
\Rightarrow \\
\left\{\begin{array}{c}
\left(u_{0}(s), \lambda\right) \in U, \\
C_{1}\left\|f\left(u_{0}(s), \lambda\right)-f\left(u_{0}(s), \lambda_{0}\right)\right\|_{\mathbb{R}^{m}} \leq \frac{1}{4} \min \left\{\delta^{\prime}, \frac{\varepsilon}{2 C_{2}}\right\}
\end{array}\right.
\end{gathered}
$$

holds. Now, we set

$$
V:=\left\{x \in \mathbb{R}^{m} \mid\left\|x-x_{0}\right\|_{\mathbb{R}^{m}}<\frac{1}{4} \min \left\{\delta^{\prime}, \frac{\varepsilon}{2 C_{2}}\right\}\right\} \times\left(\Lambda^{\prime} \cap \Lambda^{\prime \prime \prime}\right) .
$$

Then, $\left(x_{0}, \lambda_{0}\right) \in V$ holds. Also, $V$ is an open set of $\mathbb{R}^{m} \times \Lambda$. However, from $u_{0}(0)=x_{0}, A(0)=1_{\mathbb{R}^{m}}$ and (4.1), $V \subset U$ holds. $V$ is an open set of $U$.

Well, we show that (2) is established. For $v \in C\left([0,1] ; \mathbb{R}^{m}\right)$, we set

$$
\|v\|_{C\left([0,1] ; \mathbb{R}^{m}\right)}:=\sup _{s \in[0,1]}\|v(s)\|_{\mathbb{R}^{m}} .
$$

We set

$$
\tilde{X}:=\left\{v \in C\left([0,1] ; \mathbb{R}^{m}\right) \mid\|v\|_{C\left([0,1] ; \mathbb{R}^{m}\right)} \leq \min \left\{\delta^{\prime}, \frac{\varepsilon}{2 C_{2}}\right\}\right\} .
$$

$\tilde{X}$ is a non-empty complete metric space. Let $(x, \lambda) \in V$. Then, from (4.1), for any $v \in \tilde{X}$ and $s \in[0,1]$, $\left(u_{0}(s)+A(s) v(s), \lambda\right) \in U$ holds. Now, for $v \in \tilde{X}$ and $s \in[0,1]$, we set 


$$
\begin{gathered}
:=\left(x-x_{0}\right)+\int_{0}^{s}(A(\sigma))^{-1} \\
\left(f\left(u_{0}(\sigma)+A(\sigma) v(\sigma), \lambda\right)-\left(f\left(u_{0}(\sigma), \lambda_{0}\right)+\left(\left(D_{x} f\right)\left(u_{0}(\sigma), \lambda_{0}\right)\right) A(\sigma) v(\sigma)\right)\right) d \sigma .
\end{gathered}
$$

We show that $F$ is a contraction mapping of $\tilde{X}$. As $v \in \tilde{X}, s \in[0,1], \sigma \in[0,1]$ and $\tau \in[0,1]$ hold, because of

$$
\|\tau v(\sigma)\|_{\mathbb{R}^{m}} \leq \tau \min \left\{\delta^{\prime}, \frac{\varepsilon}{2 C_{2}}\right\} \leq \delta^{\prime}
$$

from (4.1) and (4.2),

$$
\begin{aligned}
& \left(u_{0}(\sigma)+\tau A(\sigma) v(\sigma), \lambda\right) \in U \\
& f\left(u_{0}(\sigma)+A(\sigma) v(\sigma), \lambda\right)-f\left(u_{0}(\sigma), \lambda\right) \\
& =\left(\int_{0}^{1}\left(D_{x} f\right)\left(u_{0}(\sigma)+\tau A(\sigma) v(\sigma), \lambda\right) d \tau\right) A(\sigma) v(\sigma), \\
& \left\|(A(\sigma))^{-1}\left(\left(f\left(u_{0}(\sigma)+A(\sigma) v(\sigma), \lambda\right)-f\left(u_{0}(\sigma), \lambda\right)\right)-\left(\left(D_{x} f\right)\left(u_{0}(\sigma), \lambda_{0}\right)\right) A(\sigma) v(\sigma)\right)\right\|_{\mathbb{R}^{n}} \\
& =\left\|(A(\sigma))^{-1}\left(\int_{0}^{1}\left(\left(D_{x} f\right)\left(u_{0}(\sigma)+\tau A(\sigma) v(\sigma), \lambda\right)-\left(D_{x} f\right)\left(u_{0}(\sigma), \lambda_{0}\right)\right) d \tau\right) A(\sigma) v(\sigma)\right\|_{\mathbb{R}^{m}} \\
& \leq \frac{1}{2}\|v(\sigma)\|_{\mathbb{R}^{m}} \leq \frac{1}{2}\|v\|_{C\left([0,1] ; \mathbb{R}^{m}\right)}, \\
& \|(F(v))(s)\|_{\mathbb{R}^{m}} \\
& \leq\left\|x-x_{0}\right\|_{\mathbb{R}^{m}}+\left\|\int_{0}^{s}(A(\sigma))^{-1}\left(f\left(u_{0}(\sigma), \lambda\right)-f\left(u_{0}(\sigma), \lambda_{0}\right)\right) d \sigma\right\|_{\mathbb{R}^{m}}+\frac{1}{2}\|v\|_{C\left([0,1] ; \mathbb{R}^{m}\right)} \\
& \leq \frac{1}{4} \min \left\{\delta^{\prime}, \frac{\varepsilon}{2 C_{2}}\right\}+\frac{1}{4} \min \left\{\delta^{\prime}, \frac{\varepsilon}{2 C_{2}}\right\}+\frac{1}{2} \min \left\{\delta^{\prime}, \frac{\varepsilon}{2 C_{2}}\right\}=\min \left\{\delta^{\prime}, \frac{\varepsilon}{2 C_{2}}\right\}
\end{aligned}
$$

hold. So, $F(\tilde{X}) \subset \tilde{X}$ holds. Next, as $v \in \tilde{X}, v^{\prime} \in \tilde{X}, s \in[0,1], \sigma \in[0,1]$ and $\tau \in[0,1]$ hold and we set

$$
c_{\tau}:=(1-\tau) v+\tau v^{\prime}=v+\tau\left(v^{\prime}-v\right),
$$

because of

$$
\left\|c_{\tau}(\sigma)\right\|_{\mathbb{R}^{m}} \leq(1-\tau) \min \left\{\delta^{\prime}, \frac{\varepsilon}{2 C_{2}}\right\}+\tau \min \left\{\delta^{\prime}, \frac{\varepsilon}{2 C_{2}}\right\} \leq \delta^{\prime}
$$

from (4.1),

$$
\begin{gathered}
\left(u_{0}(\sigma)+A(\sigma) c_{\tau}(\sigma), \lambda\right) \in U, \\
f\left(u_{0}(\sigma)+A(\sigma) v^{\prime}(\sigma), \lambda\right)-f\left(u_{0}(\sigma)+A(\sigma) v(\sigma), \lambda\right) \\
=\left(\int_{0}^{1}\left(D_{x} f\right)\left(u_{0}(\sigma)+A(\sigma) c_{\tau}(\sigma), \lambda\right) d \tau\right) A(\sigma)\left(v^{\prime}(\sigma)-v(\sigma)\right), \\
\left\|\left(F\left(v^{\prime}\right)\right)(s)-(F(v))(s)\right\|_{\mathbb{R}^{m}} \\
=\| \int_{0}^{s}(A(\sigma))^{-1} \\
\left(\left(f\left(u_{0}(\sigma)+A(\sigma) v^{\prime}(\sigma), \lambda\right)-f\left(u_{0}(\sigma)+A(\sigma) v(\sigma), \lambda\right)\right)\right. \\
\left.-\left(\left(D_{x} f\right)\left(u_{0}(\sigma), \lambda_{0}\right)\right) A(\sigma)\left(v^{\prime}(\sigma)-v(\sigma)\right)\right) d \sigma \|_{\mathbb{R}^{n}} \\
1 \\
=\int_{0}^{s}(A(\sigma))^{-1}\left(\int_{0}^{1}\left(\left(D_{x} f\right)\left(u_{0}(\sigma)+A(\sigma) c_{\tau}(\sigma), \lambda\right)-\left(D_{x} f\right)\left(u_{0}(\sigma), \lambda_{0}\right)\right) d \tau\right)
\end{gathered}
$$




$$
\begin{gathered}
A(\sigma)\left(v^{\prime}(\sigma)-v(\sigma)\right) d \sigma \|_{\mathbb{R}^{m}} \\
\quad \leq \frac{1}{2}\left\|v^{\prime}-v\right\|_{C\left([0,1] ; \mathbb{R}^{m}\right)}
\end{gathered}
$$

hold. $F$ is a contraction mapping of $\tilde{X}$. There exists $v \in \tilde{X}$ such that

$$
v=F(v)
$$

holds. We set

$$
u:=u_{0}+A v
$$

Then,

$$
\begin{gathered}
\frac{d}{d s} u=\frac{d}{d s} u_{0}+\left(\frac{d}{d s} A\right) v+A\left(\frac{d}{d s} v\right) \\
=f\left(u_{0}, \lambda_{0}\right)+\left(\left(D_{x} f\right)\left(u_{0}, \lambda_{0}\right)\right) A v+A\left(\frac{d}{d s} F(v)\right) \\
=f\left(u_{0}, \lambda_{0}\right)+\left(\left(D_{x} f\right)\left(u_{0}, \lambda_{0}\right)\right) A v+A A^{-1}\left(f(u, \lambda)-\left(f\left(u_{0}, \lambda_{0}\right)+\left(\left(D_{x} f\right)\left(u_{0}, \lambda_{0}\right)\right) A v\right)\right) \\
=f(u, \lambda), \\
u(0)=u_{0}(0)+A(0) v(0)=x_{0}+(F(v))(0)=x_{0}+\left(x-x_{0}\right)=x, \\
\left\|u-u_{0}\right\|_{C\left([0,1] ; \mathbb{R}^{m}\right)}=\|A v\|_{C\left([0,1] ; \mathbb{R}^{m}\right)} \leq C_{2} \min \left\{\delta^{\prime}, \frac{\varepsilon}{2 C_{2}}\right\} \leq \frac{\varepsilon}{2}<\varepsilon
\end{gathered}
$$

hold.

Lemma 4.10 (Holomorphic ODE) :

Let $U$ be an open set of $\mathbb{C}^{m}$. Suppose that $f: U \rightarrow \mathbb{C}^{m}$ is a holomorphic map. Let $V$ be an open set of $U$. Suppose that a map $w:[0,1] \times V \rightarrow U$ satisfies

$$
\frac{\partial w}{\partial s}(s, z)=f(w(s, z)), \quad w(0, z)=z
$$

Then, for any $s \in[0,1]$, the map

$$
z \in V \mapsto w(s, z) \in U
$$

is holomorphic.

Proof : Let $\left(x_{0}, y_{0}\right) \in V\left(\subset \mathbb{C}^{m}=\mathbb{R}^{2 m}\right)$. We denote the Fréchet derivative of $w$ for the variable $(x, y) \in V$ at a point $\left(s, x_{0}, y_{0}\right)$ by $A(s)$. That is, we set

$$
A(s):=\left(D_{(x, y)} w\right)\left(s, x_{0}, y_{0}\right) .
$$

Then, $A:[0,1] \rightarrow L\left(\mathbb{R}^{2 m} ; \mathbb{R}^{2 m}\right)$ satisfies

$$
\begin{gathered}
\frac{d}{d s} A=\left(\left(D_{(x, y)} f\right)\left(w\left(s, x_{0}, y_{0}\right)\right)\right) A, \\
A(0)=1_{\mathbb{R}^{2 m}} .
\end{gathered}
$$

On the other hand, since $f$ is holomorphic, $\left(D_{(x, y)} f\right)\left(w\left(s, x_{0}, y_{0}\right)\right)$ is complex linear. Therefore, for any $c \in \mathbb{C}$ and $(p, q) \in \mathbb{C}^{m}=\mathbb{R}^{2 m}$, both $c((A(s))(p, q))$ and $(A(s))(c(p, q))$ are the solutions of the initial value problem

$$
\begin{aligned}
\frac{d}{d s}(u, v)= & \left(\left(D_{(x, y)} f\right)\left(w\left(s, x_{0}, y_{0}\right)\right)\right)(u, v), \\
& (u, v)(0)=c(p, q)
\end{aligned}
$$

to coincide. $\left(D_{(x, y)} w\right)\left(s, x_{0}, y_{0}\right)$ is complex linear.

\section{Proposition 4.11 :}

Let $\psi \in R \subset S$. Then, $I^{R, \psi}$ is an open set of $U^{R, \psi}\left(=\mathbb{C}^{n} \times N^{R, \psi} \times W_{R}\right)$. The map $\left(\dot{e}^{R, \psi}, e^{R, \psi}\right)$ is continuous. For any $t \in \cap_{\varphi \in R} \overline{X_{\varphi}^{\prime}}$ and $c \in W_{R}$, the map $(\dot{z}, z) \mapsto\left(\dot{e}^{R, \psi}, e^{R, \psi}\right)(\dot{z}, z, t, c)$ is holomorphic. For any $(z, t) \in N^{R, \psi}$ and $c \in W_{R}$,

$$
(0, z, t, c) \in I^{R, \psi}
$$




$$
\begin{gathered}
\left(\dot{e}^{R, \psi}, e^{R, \psi}\right)(0, z, t, c)=(0, z), \\
\left(D_{\dot{z}} \dot{e}^{R, \psi}\right)(0, z, t, c)=1_{\mathbb{C}^{n}}=1_{\mathbb{R}^{2 n}}, \\
\left(D_{\dot{z}} e^{R, \psi}\right)(0, z, t, c)=1_{\mathbb{C}^{n}}=1_{\mathbb{R}^{2 n}}
\end{gathered}
$$

hold.

Proof : In virtue of Lemma 4.4 and Lemma 4.9, $I^{R, \psi}$ is an open set of $U^{R, \psi}$ and $\left(\dot{e}^{R, \psi}, e^{R, \psi}\right)$ is continuous. In virtue of Lemma 4.10, for any $t \in \cap_{\varphi \in R} \overline{X_{\varphi}^{\prime}}$ and $c \in W_{R},(\dot{z}, z) \mapsto\left(\dot{e}^{R, \psi}, e^{R, \psi}\right)(\dot{z}, z, t, c)$ is holomorphic. The others follow because $\left(\dot{e}^{R, \psi}, e^{R, \psi}\right)(\dot{z}, z, t, c)$ is the value at the time 1 of the integral curve of the spray with its value $(\dot{z}, z)$ at the time 0 .

Lemma 4.12 (Inverse function theorem with a continuous parameter) :

Let $\Lambda$ be a topological space. Let $U$ be an open set of $\mathbb{R}^{m} \times \Lambda$. Suppose that $f: U \rightarrow \mathbb{R}^{m}$ is a continuous map. Suppose that for any $\lambda \in \Lambda$, the map $x \mapsto f(x, \lambda)$ is differentiable. Suppose that the map $D_{x} f: U \rightarrow$ $L\left(\mathbb{R}^{m} ; \mathbb{R}^{m}\right)$ is continuous. Further, suppose that $\Theta$ is a compact subset of $\Lambda$. Suppose that $\{0\} \times \Theta \subset U$ and

$$
\lambda \in \Theta \Rightarrow\left(D_{x} f\right)(0, \lambda)=1_{\mathbb{R}^{m}}
$$

hold. Then, for any $\varepsilon>0$, there exist $\delta>0$, an open set $\Lambda^{\prime}$ of $\Lambda$, an open set $U^{\prime}$ of $U$ and an open set $V^{\prime}$ of $\mathbb{R}^{m} \times \Lambda$ such that they satisfy the followings.

(1)

$$
\begin{gathered}
\Theta \subset \Lambda^{\prime}, \\
\left\{x \in \mathbb{R}^{m} \mid\|x\|_{\mathbb{R}^{m}}<\delta\right\} \times \Lambda^{\prime} \subset U^{\prime} \subset \mathbb{R}^{m} \times \Lambda^{\prime}, \\
\cup_{\lambda \in \Lambda^{\prime}}\left(\left\{y \in \mathbb{R}^{m} \mid\|y-f(0, \lambda)\|_{\mathbb{R}^{m}}<\delta\right\} \times\{\lambda\}\right) \subset V^{\prime} \subset \mathbb{R}^{m} \times \Lambda^{\prime}, \\
\sup _{(x, \lambda) \in U^{\prime}}\left\|\left(D_{x} f\right)(x, \lambda)-1_{\mathbb{R}^{m}}\right\|_{L\left(\mathbb{R}^{m} ; \mathbb{R}^{m}\right)} \leq \varepsilon
\end{gathered}
$$

hold. Homeomorphically, the map $(x, \lambda) \in U \mapsto(f(x, \lambda), \lambda) \in \mathbb{R}^{m} \times \Lambda$ maps $U^{\prime}$ to $V^{\prime}$.

(2) There exists a continuous map $g: V^{\prime} \rightarrow \mathbb{R}^{m}$ such that it satisfies the followings. For any $(y, \lambda) \in V^{\prime}$,

$$
(g(y, \lambda), \lambda) \in U^{\prime}, f(g(y, \lambda), \lambda)=y
$$

hold. For any $\lambda \in \Lambda^{\prime}$, the map $y \mapsto g(y, \lambda)$ is differentiable. The map $D_{y} g: V^{\prime} \rightarrow L\left(\mathbb{R}^{m} ; \mathbb{R}^{m}\right)$ is continuous.

$$
\sup _{(y, \lambda) \in V^{\prime}}\left\|\left(D_{y} g\right)(y, \lambda)-1_{\mathbb{R}^{m}}\right\|_{L\left(\mathbb{R}^{m} ; \mathbb{R}^{m}\right)} \leq \varepsilon
$$

holds.

Proof : This is the same as proofs of usual inverse function theorems. Just to be sure, we confirm it.

When $\Theta=\emptyset$ holds, it is trivial. Let $\Theta \neq \emptyset$ and $\varepsilon>0$. We set

$$
\varepsilon^{\prime}:=\frac{\varepsilon}{1+\varepsilon} \text {. }
$$

$0<\varepsilon^{\prime}<\min \{\varepsilon, 1\}$ holds. For $\lambda \in \Theta$, there exist $\delta_{\lambda}^{\prime \prime}>0$ and an open set $\Lambda_{\lambda}^{\prime \prime}$ of $\Lambda$ such that $\lambda \in \Lambda_{\lambda}^{\prime \prime}$ and

$$
\begin{gathered}
x \in \mathbb{R}^{m},\|x\|_{\mathbb{R}^{m}} \leq \delta_{\lambda}^{\prime \prime}, \lambda^{\prime \prime} \in \Lambda_{\lambda}^{\prime \prime} \\
\Rightarrow \quad\left(x, \lambda^{\prime \prime}\right) \in U,\left\|\left(D_{x} f\right)\left(x, \lambda^{\prime \prime}\right)-1_{\mathbb{R}^{m}}\right\|_{L\left(\mathbb{R}^{m} ; \mathbb{R}^{m}\right)} \leq \varepsilon^{\prime}
\end{gathered}
$$

hold. Then, there exists a finite subset $L(\neq \emptyset)$ of $\Theta$ such that

$$
\Theta \subset \Lambda^{\prime}:=\cup_{\lambda \in L} \Lambda_{\lambda}^{\prime \prime}
$$

holds. $\Lambda^{\prime}$ is an open set of $\Lambda$. Now, we set

$$
\begin{gathered}
\delta^{\prime}:=\min _{\lambda \in L} \delta_{\lambda}^{\prime \prime}, \\
E:=\left\{x \in \mathbb{R}^{m} \mid\|x\|_{\mathbb{R}^{m}} \leq \delta^{\prime}\right\} \times \Lambda^{\prime} .
\end{gathered}
$$


$\delta^{\prime}>0$ holds. Also, as $(x, \lambda) \in E$ holds, because there exists $\lambda^{\prime \prime} \in L$ such that $\lambda \in \Lambda_{\lambda^{\prime \prime}}^{\prime \prime}$ holds, from $\|x\|_{\mathbb{R}^{m}} \leq \delta^{\prime} \leq$ $\delta_{\lambda^{\prime \prime}}^{\prime \prime},(x, \lambda) \in U$ and $\left\|\left(D_{x} f\right)(x, \lambda)-1_{\mathbb{R}^{m}}\right\|_{L\left(\mathbb{R}^{m} ; \mathbb{R}^{m}\right)} \leq \varepsilon^{\prime}$ hold. So,

$$
\begin{gathered}
\{0\} \times \Lambda^{\prime} \subset E \subset U, \\
\sup _{(x, \lambda) \in E}\left\|1_{\mathbb{R}^{m}}-\left(D_{x} f\right)(x, \lambda)\right\|_{L\left(\mathbb{R}^{m} ; \mathbb{R}^{m}\right)} \leq \varepsilon^{\prime}
\end{gathered}
$$

hold. Also, we set

$$
\begin{gathered}
E^{\prime}:=\cup_{\lambda \in \Lambda^{\prime}}\left(\left\{y \in \mathbb{R}^{m} \mid\|y-f(0, \lambda)\|_{\mathbb{R}^{m}} \leq\left(1-\varepsilon^{\prime}\right) \delta^{\prime}\right\} \times\{\lambda\}\right), \\
E^{\prime \prime}:=\left\{x \in \mathbb{R}^{m} \mid\|x\|_{\mathbb{R}^{m}} \leq \delta^{\prime}\right\} \times E^{\prime} .
\end{gathered}
$$

Then,

$$
\begin{gathered}
(x, \lambda) \in E \quad \Rightarrow \quad(x, f(0, \lambda), \lambda) \in E^{\prime \prime}, \\
(x, y, \lambda) \in E^{\prime \prime} \quad \Rightarrow \quad(x, \lambda) \in E
\end{gathered}
$$

hold. We define a map $h: E^{\prime \prime} \rightarrow \mathbb{R}^{m}$ as

$$
h(x, y, \lambda):=x-f(x, \lambda)+y .
$$

As $(x, y, \lambda) \in E^{\prime \prime}$ and $s \in[0,1]$ hold, $(s x, \lambda) \in E$ and

$$
\begin{gathered}
h(x, y, \lambda)=((x-f(x, \lambda))-(0-f(0, \lambda)))+(y-f(0, \lambda)) \\
=\left(\int_{0}^{1}\left(1_{\mathbb{R}^{m}}-\left(D_{x} f\right)(s x, \lambda)\right) d s\right) x+(y-f(0, \lambda))
\end{gathered}
$$

hold. Hence, by (4.3),

$$
\begin{gathered}
(x, y, \lambda) \in E^{\prime \prime} \\
\Rightarrow \\
\|h(x, y, \lambda)\|_{\mathbb{R}^{m}} \leq \varepsilon^{\prime}\|x\|_{\mathbb{R}^{m}}+\|y-f(0, \lambda)\|_{\mathbb{R}^{m}} \leq \delta^{\prime}
\end{gathered}
$$

holds. As $(x, y, \lambda) \in E^{\prime \prime},\left(x^{\prime}, y, \lambda\right) \in E^{\prime \prime}$ and $s \in[0,1]$ hold and we set $c(s):=(1-s) x+s x^{\prime}=x+s\left(x^{\prime}-x\right)$, $(c(s), \lambda) \in E$ and

$$
\begin{gathered}
h\left(x^{\prime}, y, \lambda\right)-h(x, y, \lambda)=\left(x^{\prime}-f\left(x^{\prime}, \lambda\right)\right)-(x-f(x, \lambda)) \\
=\left(\int_{0}^{1}\left(1_{\mathbb{R}^{m}}-\left(D_{x} f\right)(c(s), \lambda)\right) d s\right)\left(x^{\prime}-x\right)
\end{gathered}
$$

hold. Hence, by (4.3),

$$
\begin{gathered}
(x, y, \lambda) \in E^{\prime \prime},\left(x^{\prime}, y, \lambda\right) \in E^{\prime \prime} \\
\Rightarrow \\
\left\|h\left(x^{\prime}, y, \lambda\right)-h(x, y, \lambda)\right\|_{\mathbb{R}^{m}} \leq \varepsilon^{\prime}\left\|x^{\prime}-x\right\|_{\mathbb{R}^{m}}
\end{gathered}
$$

holds.

For $\ell \in C\left(E^{\prime} ; \mathbb{R}^{m}\right)$, we set

$$
\|\ell\|_{C\left(E^{\prime} ; \mathbb{R}^{m}\right)}:=\sup _{(y, \lambda) \in E^{\prime}}\|\ell(y, \lambda)\|_{\mathbb{R}^{m}}
$$

We set

$$
\tilde{X}:=\left\{\ell \in C\left(E^{\prime} ; \mathbb{R}^{m}\right) \mid\|\ell\|_{C\left(E^{\prime} ; \mathbb{R}^{m}\right)} \leq \delta^{\prime}\right\} .
$$

$\tilde{X}$ is a non-empty complete metric space. For any $\ell \in \tilde{X}$ and $(y, \lambda) \in E^{\prime},(\ell(y, \lambda), y, \lambda) \in E^{\prime \prime}$ holds. Now, for $\ell \in \tilde{X}$ and $(y, \lambda) \in E^{\prime}$, we set

$$
(F(\ell))(y, \lambda):=h(\ell(y, \lambda), y, \lambda) .
$$


As $\ell \in \tilde{X}$ holds, because of $F(\ell) \in C\left(E^{\prime} ; \mathbb{R}^{m}\right)$, from (4.4), $F(\ell) \in \tilde{X}$ holds. Further, as $\ell \in \tilde{X}$ and $\ell^{\prime} \in \tilde{X}$ hold, from (4.5), $\left\|F\left(\ell^{\prime}\right)-F(\ell)\right\|_{C\left(E^{\prime} ; \mathbb{R}^{m}\right)} \leq \varepsilon^{\prime}\left\|\ell^{\prime}-\ell\right\|_{C\left(E^{\prime} ; \mathbb{R}^{m}\right)}$ holds. $F$ is a contraction mapping of $\tilde{X}$. Therefore, there exists $\ell \in \tilde{X}$ such that

$$
\ell=F(\ell)
$$

holds. In particular,

$$
(y, \lambda) \in E^{\prime} \quad \Rightarrow \quad(\ell(y, \lambda), \lambda) \in E, f(\ell(y, \lambda), \lambda)=y
$$

holds.

Now, we set

$$
\begin{gathered}
V^{\prime}:=\cup_{\lambda \in \Lambda^{\prime}}\left(\left\{y \in \mathbb{R}^{m} \mid\|y-f(0, \lambda)\|_{\mathbb{R}^{m}}<\left(1-\varepsilon^{\prime}\right) \delta^{\prime}\right\} \times\{\lambda\}\right), \\
g:=\ell_{\mid V^{\prime}}, \\
U^{\prime}:=\left\{(g(y, \lambda), \lambda) \in E \mid(y, \lambda) \in V^{\prime}\right\} .
\end{gathered}
$$

$V^{\prime}$ is an open set of $\mathbb{R}^{m} \times \Lambda . g \in C\left(V^{\prime} ; \mathbb{R}^{m}\right)$ and

$$
(y, \lambda) \in V^{\prime} \quad \Rightarrow \quad(g(y, \lambda), \lambda) \in U^{\prime}, f(g(y, \lambda), \lambda)=y
$$

hold. On the other hand, as $(x, \lambda) \in U^{\prime}$ holds, because there exists $y \in \mathbb{R}^{m}$ such that $(y, \lambda) \in V^{\prime}$ and $x=g(y, \lambda)$ hold,

$$
\begin{gathered}
y=f(g(y, \lambda), \lambda)=f(x, \lambda), \\
(f(x, \lambda), \lambda)=(y, \lambda) \in V^{\prime}, \\
x=g(y, \lambda)=g(f(x, \lambda), \lambda)
\end{gathered}
$$

hold. That is,

$$
(x, \lambda) \in U^{\prime} \quad \Rightarrow \quad(f(x, \lambda), \lambda) \in V^{\prime}, g(f(x, \lambda), \lambda)=x
$$

holds. Therefore, homeomorphically the map $(x, \lambda) \mapsto(f(x, \lambda), \lambda)$ maps $U^{\prime}$ to $V^{\prime}$.

Also, from (4.4), for any $(y, \lambda) \in V^{\prime},\|g(y, \lambda)\|_{\mathbb{R}^{m}}=\|\ell(y, \lambda)\|_{\mathbb{R}^{m}}=\|(F(\ell))(y, \lambda)\|_{\mathbb{R}^{m}}=$ $\|h(\ell(y, \lambda), y, \lambda)\|_{\mathbb{R}^{m}} \leq \varepsilon^{\prime} \delta^{\prime}+\|y-f(0, \lambda)\|_{\mathbb{R}^{m}}<\varepsilon^{\prime} \delta^{\prime}+\left(1-\varepsilon^{\prime}\right) \delta^{\prime}=\delta^{\prime}$ holds. Therefore,

$$
U^{\prime} \subset G:=\left\{x \in \mathbb{R}^{m} \mid\|x\|_{\mathbb{R}^{m}}<\delta^{\prime}\right\} \times \Lambda^{\prime} \subset E \subset U
$$

holds. So,

$$
(x, \lambda) \in U^{\prime} \quad \Rightarrow \quad(x, \lambda) \in G,(f(x, \lambda), \lambda) \in V^{\prime}
$$

holds. Conversely, if $(x, \lambda) \in G$ and $(f(x, \lambda), \lambda) \in V^{\prime}$ hold, then 


$$
\begin{gathered}
g(f(x, \lambda), \lambda)=\ell(f(x, \lambda), \lambda)=(F(\ell))(f(x, \lambda), \lambda) \\
=h(\ell(f(x, \lambda), \lambda), f(x, \lambda), \lambda)=h(g(f(x, \lambda), \lambda), f(x, \lambda), \lambda), \\
x=h(x, f(x, \lambda), \lambda)
\end{gathered}
$$

hold and so, from (4.5),

$$
\begin{gathered}
\|g(f(x, \lambda), \lambda)-x\|_{\mathbb{R}^{m}} \\
\leq\|h(g(f(x, \lambda), \lambda), f(x, \lambda), \lambda)-h(x, f(x, \lambda), \lambda)\|_{\mathbb{R}^{m}} \\
\leq \varepsilon^{\prime}\|g(f(x, \lambda), \lambda)-x\|_{\mathbb{R}^{m}}, \\
g(f(x, \lambda), \lambda)=x, \\
(x, \lambda)=(g(f(x, \lambda), \lambda), \lambda) \in U^{\prime}
\end{gathered}
$$

hold. Thus,

$$
\begin{aligned}
(x, \lambda) \in G, \quad(f(x, \lambda), \lambda) \in V^{\prime} \\
\Longleftrightarrow \quad(x, \lambda) \in U^{\prime}
\end{aligned}
$$

holds. That is, $U^{\prime}=\left\{(x, \lambda) \in G \mid(f(x, \lambda), \lambda) \in V^{\prime}\right\}$ holds. $U^{\prime}$ is an open set of $U$.

Further, we set

$$
\delta:=\frac{1-\varepsilon^{\prime}}{1+\varepsilon^{\prime}} \delta^{\prime}
$$

$\delta>0$ and

$$
\cup_{\lambda \in \Lambda^{\prime}}\left(\left\{y \in \mathbb{R}^{m} \mid\|y-f(0, \lambda)\|_{\mathbb{R}^{m}}<\delta\right\} \times\{\lambda\}\right) \subset V^{\prime} \subset \mathbb{R}^{m} \times \Lambda^{\prime}
$$

hold. As $x \in \mathbb{R}^{m},\|x\|_{\mathbb{R}^{m}}<\delta, \lambda \in \Lambda^{\prime}$ and $s \in[0,1]$ hold, because of (sx, $\left.\lambda\right) \in G \subset E$, from (4.3) and (4.6), $\|f(x, \lambda)-f(0, \lambda)\|_{\mathbb{R}^{m}} \leq\left(1+\varepsilon^{\prime}\right)\|x\|_{\mathbb{R}^{m}}<\left(1+\varepsilon^{\prime}\right) \delta=\left(1-\varepsilon^{\prime}\right) \delta^{\prime},(f(x, \lambda), \lambda) \in V^{\prime}$ and $(x, \lambda) \in U^{\prime}$ hold. Therefore,

$$
\left\{x \in \mathbb{R}^{m} \mid\|x\|_{\mathbb{R}^{m}}<\delta\right\} \times \Lambda^{\prime} \subset U^{\prime} \subset \mathbb{R}^{m} \times \Lambda^{\prime}
$$

holds.

From (4.3),

$$
\sup _{(x, \lambda) \in U^{\prime}}\left\|\left(D_{x} f\right)(x, \lambda)-1_{\mathbb{R}^{m}}\right\|_{L\left(\mathbb{R}^{m} ; \mathbb{R}^{m}\right)} \leq \varepsilon^{\prime}<\min \{\varepsilon, 1\}
$$

holds. From a usual inverse function theorem, for any $\lambda \in \Lambda^{\prime}$, the map $y \mapsto g(y, \lambda)$ is differentiable. Hence, for any $(y, \lambda) \in V^{\prime}$,

$$
\left(D_{y} g\right)(y, \lambda)=\left(\left(D_{x} f\right)(g(y, \lambda), \lambda)\right)^{-1}
$$

holds. The map $D_{y} g: V^{\prime} \rightarrow L\left(\mathbb{R}^{m} ; \mathbb{R}^{m}\right)$ is continuous. Also, 


$$
\begin{gathered}
\sup _{(y, \lambda) \in V^{\prime}}\left\|\left(D_{y} g\right)(y, \lambda)-1_{\mathbb{R}^{m}}\right\|_{L\left(\mathbb{R}^{m} ; \mathbb{R}^{m}\right)} \\
=\sup _{(x, \lambda) \in U^{\prime}}\left\|\left(\left(D_{x} f\right)(x, \lambda)\right)^{-1}-1_{\mathbb{R}^{m}}\right\|_{L\left(\mathbb{R}^{m} ; \mathbb{R}^{m}\right)} \\
=\sup _{(x, \lambda) \in U^{\prime}}\left\|\sum_{k=1}^{\infty}\left(1_{\mathbb{R}^{m}}-\left(D_{x} f\right)(x, \lambda)\right)^{k}\right\|_{L\left(\mathbb{R}^{m} ; \mathbb{R}^{m}\right)} \\
\leq \sum_{k=1}^{\infty} \varepsilon^{\prime k}=\varepsilon
\end{gathered}
$$

holds.

\section{Definition 4.13 :}

(1) Let $R$ be a non-empty subset of $S$. Let

$$
K^{R}:=\cap_{\varphi \in R}\left(\varphi,\left.\pi\right|_{\varphi}\right)^{-1}\left(\overline{D_{r_{0}}^{n}} \times \overline{X_{\varphi}^{\prime}}\right) .
$$

$K^{R}$ is a compact subset of $N^{R}$. Further, let $\psi \in R$ and

$$
K^{R, \psi}:=\left(\psi,\left.\pi\right|_{\psi}\right)\left(K^{R}\right) .
$$

$K^{R, \psi}$ is a compact subset of $N^{R, \psi}$.

(2) Let

$$
\mathcal{P}^{\prime}(S):=\{(R, \psi) \mid \psi \in R \subset S\} .
$$

\section{Lemma 4.14 :}

There exist $\alpha_{0}>0, \beta_{0}>0, \delta_{0}^{a}>0, \delta_{0}^{b}>0$ and $\left\{\left(U_{R, \psi}^{\prime}, V_{R, \psi}^{\prime}, h^{R, \psi}\right)\right\}_{(R, \psi) \in \mathcal{P}^{\prime}(S)}$ such that for any $(R, \psi) \in$ $\mathcal{P}^{\prime}(S)$, they satisfy the followings.

(1) $U_{R, \psi}^{\prime} \subset I^{R, \psi}$ holds. $U_{R, \psi}^{\prime}$ is an open set of $\mathbb{C}^{n} \times K^{R, \psi} \times W_{R} \cdot V_{R, \psi}^{\prime}$ is an open set of $D_{\frac{r_{0}+1}{2}}^{n} \times K^{R, \psi} \times W_{R}$.

$$
\begin{gathered}
\left\{\dot{z} \in \mathbb{C}^{n} \mid\|\dot{z}\|_{\mathbb{C}^{n}}<\delta_{0}^{a}\right\} \times K^{R, \psi} \times W_{R} \subset U_{R, \psi}^{\prime}, \\
\left(\cup_{(z, t) \in K^{R, \psi}}\left(\left\{w \in \mathbb{C}^{n} \mid\|w-z\|_{\mathbb{C}^{n}}<\delta_{0}^{b}\right\} \times\{(z, t)\}\right)\right) \times W_{R} \subset \quad V_{R, \psi}^{\prime}, \\
\sup _{(\dot{z}, z, t, c) \in U_{R, \psi}^{\prime}}\left\|\left(D_{\dot{z}} e^{R, \psi}\right)(\dot{z}, z, t, c)\right\|_{L\left(\mathbb{C}^{n} ; \mathbb{C}^{n}\right)} \leq \alpha_{0}
\end{gathered}
$$

hold. Homeomorphically, the map $(\dot{z}, z, t, c) \in I^{R, \psi} \mapsto\left(e^{R, \psi}(\dot{z}, z, t, c), z, t, c\right) \in D_{\frac{r_{0}+1}{2}}^{n} \times N^{R, \psi} \times W_{R} \operatorname{maps}$ $U_{R, \psi}^{\prime}$ to $V_{R, \psi}^{\prime}$.

(2) $h^{R, \psi}$ is a continuous map from $V_{R, \psi}^{\prime}$ to $\mathbb{C}^{n}$. For any $(w, z, t, c) \in V_{R, \psi}^{\prime}$,

$$
\begin{gathered}
\left(h^{R, \psi}(w, z, t, c), z, t, c\right) \in U_{R, \psi}^{\prime}, \\
e^{R, \psi}\left(h^{R, \psi}(w, z, t, c), z, t, c\right)=w
\end{gathered}
$$

hold. For any $(z, t) \in K^{R, \psi}$ and $c \in W_{R}$, the map $w \mapsto h^{R, \psi}(w, z, t, c)$ is holomorphic.

$$
\sup _{(w, z, t, c) \in V_{R, \psi}^{\prime}}\left\|\left(D_{w} h^{R, \psi}\right)(w, z, t, c)\right\|_{L\left(\mathbb{C}^{n} ; \mathbb{C}^{n}\right)} \leq \beta_{0}
$$

holds.

Proof : Since $\mathcal{P}^{\prime}(S)$ is a finite set, it is sufficient to discuss it for each $(R, \psi) \in \mathcal{P}^{\prime}(S)$. Let $(R, \psi) \in \mathcal{P}^{\prime}(S)$. As we set

$$
\begin{gathered}
\Theta:=\Lambda:=K^{R, \psi} \times W_{R}, \\
U:=I^{R, \psi} \cap\left(\mathbb{C}^{n} \times K^{R, \psi} \times W_{R}\right)=I^{R, \psi} \cap\left(\mathbb{R}^{2 n} \times \Lambda\right),
\end{gathered}
$$




$$
f:=e^{R, \psi}{ }_{\lceil U},
$$

in virtue of Lemma 1.11 and Proposition 4.11, we can apply Lemma 4.12. lemma.

Hereafter, we fix $\alpha_{0}>0, \beta_{0}>0, \delta_{0}^{a}>0, \delta_{0}^{b}>0$ and $\left\{\left(U_{R, \psi}^{\prime}, V_{R, \psi}^{\prime}, h^{R, \psi}\right)_{(R, \psi) \in \mathcal{P}^{\prime}(S)}\right.$ given by the above

\section{Definition 4.15 :}

Let $\left\{\left(U_{R, \psi}^{\prime}, V_{R, \psi}^{\prime}, h^{R, \psi}\right)\right\}_{(R, \psi) \in \mathcal{P}^{\prime}(S)}$ be the same as Lemma 4.14. Let $\psi \in R \subset S$. Further, suppose that $T^{\prime}$ is a subset of $\overline{X_{\psi}^{\prime}}, \chi^{\prime}$ is a continuous map from $T^{\prime}$ to $W_{R}$ and $v^{\prime}$ is a continuous map from $T^{\prime}$ to $\overline{D_{r_{0}}^{n}}$ such that for any $t \in T^{\prime},\left(v^{\prime}(t), t\right) \in K^{R, \psi}$ holds. Then, let

$$
\begin{aligned}
U_{R, \psi, v^{\prime}, \chi^{\prime}}^{\prime} & :=\left\{(\dot{z}, t) \in \mathbb{C}^{n} \times T^{\prime} \mid\left(\dot{z}, v^{\prime}(t), t, \chi^{\prime}(t)\right) \in U_{R, \psi}^{\prime}\right\}, \\
V_{R, \psi, v^{\prime}, \chi^{\prime}}^{\prime} & :=\left\{(w, t) \in D_{\frac{r_{0}+1}{2}}^{n} \times T^{\prime} \mid\left(w, v^{\prime}(t), t, \chi^{\prime}(t)\right) \in V_{R, \psi}^{\prime}\right\} .
\end{aligned}
$$

Further, a map $e^{R, \psi, v^{\prime}, \chi^{\prime}}$ from $U_{R, \psi, v^{\prime}, \chi^{\prime}}^{\prime}$ to $D_{\frac{r_{0}+1}{2}}^{n}$ is defined as

$$
e^{R, \psi, v^{\prime}, \chi^{\prime}}(\dot{z}, t):=e^{R, \psi}\left(\dot{z}, v^{\prime}(t), t, \chi^{\prime}(t)\right)
$$

and a map $h^{R, \psi, v^{\prime}, \chi^{\prime}}$ from $V_{R, \psi, v^{\prime}, \chi^{\prime}}^{\prime}$ to $\mathbb{C}^{n}$ is defined as

$$
h^{R, \psi, v^{\prime}, \chi^{\prime}}(w, t):=h^{R, \psi}\left(w, v^{\prime}(t), t, \chi^{\prime}(t)\right) .
$$

\section{Lemma 4.16 :}

Let $\alpha_{0}>0, \beta_{0}>0, \delta_{0}^{a}>0$ and $\delta_{0}^{b}>0$ be the same as Lemma 4.14. Let $\psi \in R \subset S$. Further, suppose that $T^{\prime}$ is a subset of $\overline{X_{\psi}^{\prime}}, \chi^{\prime}$ is a continuous function from $T^{\prime}$ to $W_{R}$ and $v^{\prime}$ is a continuous function from $T^{\prime}$ to $\overline{D_{r_{0}}^{n}}$ such that for any $t \in T^{\prime},\left(v^{\prime}(t), t\right) \in K^{R, \psi}$ holds. Then, the followings hold.

$U_{R, \psi, v^{\prime}, \chi^{\prime}}^{\prime}$ is an open set of $\mathbb{C}^{n} \times T^{\prime} . V_{R, \psi, v^{\prime}, \chi^{\prime}}^{\prime}$ is an open set of $D_{\frac{r_{0}+1}{2}}^{n} \times T^{\prime}$.

$$
\begin{gathered}
\left\{\dot{z} \in \mathbb{C}^{n} \mid\|\dot{z}\|_{\mathbb{C}^{n}}<\delta_{0}^{a}\right\} \times T^{\prime} \subset U_{R, \psi, v^{\prime}, \chi^{\prime}}^{\prime}, \\
\cup_{t \in T^{\prime}}\left(\left\{w \in \mathbb{C}^{n} \mid\left\|w-v^{\prime}(t)\right\|_{\mathbb{C}^{n}}<\delta_{0}^{b}\right\} \times\{t\}\right) \subset V_{R, \psi, v^{\prime}, \chi^{\prime}}^{\prime}
\end{gathered}
$$

hold. For any $t \in T^{\prime}$, the maps $\dot{z} \mapsto e^{R, \psi, v^{\prime}, \chi^{\prime}}(\dot{z}, t)$ and $w \mapsto h^{R, \psi, v^{\prime}, \chi^{\prime}}(w, t)$ are holomorphic.

$$
\begin{aligned}
& \sup _{(\dot{z}, t) \in U_{R, \psi, v^{\prime}, \chi^{\prime}}^{\prime}}\left\|\left(D_{\dot{z}} e^{R, \psi, v^{\prime}, \chi^{\prime}}\right)(\dot{z}, t)\right\|_{L\left(\mathbb{C}^{n} ; \mathbb{C}^{n}\right)} \leq \alpha_{0}, \\
& \sup _{(w, t) \in V_{R, \psi, v^{\prime}, \chi^{\prime}}^{\prime}}\left\|\left(D_{w} h^{R, \psi, v^{\prime}, \chi^{\prime}}\right)(w, t)\right\|_{L\left(\mathbb{C}^{n} ; \mathbb{C}^{n}\right)} \leq \beta_{0}
\end{aligned}
$$

hold. Homeomorphically, the map $(\dot{z}, t) \mapsto\left(e^{R, \psi, v^{\prime}, \chi^{\prime}}(\dot{z}, t), t\right)$ maps $U_{R, \psi, v^{\prime}, \chi^{\prime}}^{\prime}$ to $V_{R, \psi, v^{\prime}, \chi^{\prime}}^{\prime}$. For any $(w, t) \in$ $V_{R, \psi, v^{\prime}, \chi^{\prime}}^{\prime}$

$$
\begin{aligned}
& \left(h^{R, \psi, v^{\prime}, \chi^{\prime}}(w, t), t\right) \in U_{R, \psi, v^{\prime}, \chi^{\prime}}^{\prime}, \\
& e^{R, \psi, v^{\prime}, \chi^{\prime}}\left(h^{R, \psi, v^{\prime}, \chi^{\prime}}(w, t), t\right)=w
\end{aligned}
$$

hold.

Proof : It follows from Lemma 4.14.

Lemma 4.17 :

There exist $C_{1}^{a}>0$ and $C_{1}^{b}>0$ such that the following holds. For any $\varphi \in S, p \in\left(\varphi,\left.\pi\right|_{\varphi}\right)^{-1}\left(\overline{D_{\frac{r_{0}+1}{2}}^{n}} \times \overline{X_{\varphi}^{\prime}}\right)$ and $\dot{p} \in\left(T_{\mathbb{R}}\right)_{p}\left(F_{p}\right)$,

$$
\|\dot{p}\|_{\varphi, p} \leq C_{1}^{a}\|\dot{p}\|_{p}, \quad\|\dot{p}\|_{p} \leq C_{1}^{b}\|\dot{p}\|_{\varphi, p}
$$

hold. 
Proof : Since $S$ is a finite set, it follows from Lemma 1.14.

Lemma 4.18 :

There exist $\delta_{2}>0$ and $C_{2}>0$ such that the following holds. For any $\varphi \in S, p \in\left(\varphi,\left.\pi\right|_{\varphi}\right)^{-1}\left(\overline{D_{\frac{r_{0}+1}{2}}^{n}} \times \overline{X_{\varphi}^{\prime}}\right)$ and $q \in F_{p}$,

$$
\begin{gathered}
d_{\pi(p)}(q, p)<\delta_{2} \\
\Rightarrow \\
q \in M_{\varphi}, \quad\|\varphi(q)-\varphi(p)\|_{\mathbb{C}^{n}} \leq C_{2} d_{\pi(p)}(q, p)
\end{gathered}
$$

holds.

Proof : Since $S$ is a finite set, it follows from Lemma 1.16.

Proof of Proposition 3.5 :

$0^{\circ}: \quad$ Let $\alpha_{0}, \beta_{0}, \delta_{0}^{a}, \delta_{0}^{b}, \delta_{2}, C_{1}^{a}, C_{1}^{b}, C_{2}$ and $\left\{\left(U_{R, \psi}^{\prime}, V_{R, \psi}^{\prime}, h^{R, \psi}\right)\right\}_{(R, \psi) \in \mathcal{P}^{\prime}(S)}$ be the same as Lemma 4.14, Lemma 4.17 and Lemma 4.18.

In order to define a holomorphic linear spray for each $t \in T$, we first select a $t$-dependent weighting (i.e., a partition of unity) $\chi$ as follows. Because $\left\{M_{\varphi}^{\prime}\right\}_{\varphi \in S}$ is a finite open covering of $M,\left\{u^{-1}\left(M_{\varphi}^{\prime}\right)\right\}_{\varphi \in S}$ is a finite open covering of $T$. Because $T$ is normal, there exists $\chi\left(:=\left\{\chi_{\varphi}(t)\right\}_{\varphi \in S, t \in T}\right)$ such that $\chi_{\varphi}$ is a non-negative continuous function on $T$ and

$$
\operatorname{supp}\left(\chi_{\varphi}\right) \subset u^{-1}\left(M_{\varphi}^{\prime}\right), \quad \sum_{\varphi \in S} \chi_{\varphi}=1
$$

hold. 
$1^{\circ}:$ For $t \in T$, we define

$$
R_{t}:=\left\{\varphi \in S \mid t \in \operatorname{supp}\left(\chi_{\varphi}\right)\right\}
$$

Then,

$$
\begin{gathered}
\emptyset \neq R_{t} \subset S, \\
\chi(t)\left(:=\left\{\chi_{\varphi}(t)\right\}_{\varphi \in S}\right) \in W_{R_{t}}, \\
u(t) \in \cap_{\varphi \in R_{t}} M_{\varphi}^{\prime}=\cap_{\varphi \in R_{t}}\left(\varphi,\left.\pi\right|_{\varphi}\right)^{-1}\left(D_{r_{0}}^{n} \times X_{\varphi}^{\prime}\right) \subset K^{R_{t}}
\end{gathered}
$$

hold.

$2^{\circ}:$ For $p \in M$, we define

$$
B_{p}^{\prime}\left(T_{\mathbb{R}}\right):=\left\{\dot{p} \in\left(T_{\mathbb{R}}\right)_{p}\left(F_{p}\right) \mid\|\dot{p}\|_{p}<\frac{\min \left\{\delta_{0}^{a}, \frac{\delta_{0}^{b}}{\alpha_{0}}\right\}}{C_{1}^{a}}\right\} .
$$

For $\dot{p} \in \cup_{p \in u(T)} B_{p}^{\prime}\left(T_{\mathbb{R}}\right)$, we define $\exp (\dot{p}) \in \pi^{-1}(T)$ as follows.

Let $\dot{p} \in \cup_{p \in u(T)} B_{p}^{\prime}\left(T_{\mathbb{R}}\right)$. Then, there uniquely exists $t \in T$ such that $\dot{p} \in B_{u(t)}^{\prime}\left(T_{\mathbb{R}}\right)$ holds. In virtue of $1^{\circ}$, there exists $(R, \psi) \in \mathcal{P}^{\prime}(S)$ such that

$$
\chi(t) \in W_{R}, \quad u(t) \in K^{R}
$$

hold. Because of $K^{R} \subset\left(\psi,\left.\pi\right|_{\psi}\right)^{-1}\left(\overline{D_{r_{0}}^{n}} \times \overline{X_{\psi}^{\prime}}\right)$, by Lemma 4.17,

$$
\left\|\left(D\left(\left.\psi\right|_{t}\right)\right)_{u(t)}(\dot{p})\right\|_{\mathbb{C}^{n}} \leq C_{1}^{a}\|\dot{p}\|_{u(t)}<\min \left\{\delta_{0}^{a}, \frac{\delta_{0}^{b}}{\alpha_{0}}\right\}
$$

holds. Therefore, from Lemma 4.14,

$$
\left((D(\psi \mid t))_{u(t)}(\dot{p}), \psi(u(t)), t, \chi(t)\right) \in U_{R, \psi}^{\prime}
$$

holds. Hence, $\dot{p}$ belongs to the domain of the exponential map for the spray $Y_{R}^{\chi(t), t}$ on $N_{t}^{R}$. So, further, it does to the one for $Y^{\chi(t), t}$. Now, we define $\exp (\dot{p})$ as the value of the exponential map for the spray $Y^{\chi(t), t}$ at $\dot{p}$. Then,

$$
\exp (\dot{p}) \in \pi^{-1}(\{t\}) \subset \pi^{-1}(T)
$$

holds.

$3^{\circ}$ : We define

$$
U:=\exp \left(\cup_{p \in u(T)} B_{p}^{\prime}\left(T_{\mathbb{R}}\right)\right)
$$

$U$ is a subset of $\pi^{-1}(T)$. exp is a map whose domain is $\cup_{p \in u(T)} B_{p}^{\prime}\left(T_{\mathbb{R}}\right)$ and whose range is $U$.

$4^{\circ}:$ For $t_{0} \in T$, we define

$$
T_{t_{0}}:=\left(\cap_{\psi \in R_{t_{0}}}\left(u^{-1}\left(M_{\psi}^{\prime}\right)\right)\right) \cap\left(\cap_{\psi \in S \backslash R_{t_{0}}}\left(T \backslash \operatorname{supp}\left(\chi_{\psi}\right)\right)\right) .
$$

$T_{t_{0}}$ is an open set of $T$. $t_{0} \in T_{t_{0}}$ holds. Thus, in particular, $\left\{T_{t_{0}}\right\}_{t_{0} \in T}$ is an open covering of $T$. Also, 


$$
\begin{gathered}
t \in T_{t_{0}} \\
\Rightarrow \quad \chi(t) \in W_{R_{t_{0}}}, \quad u(t) \in \cap_{\varphi \in R_{t_{0}}} M_{\varphi}^{\prime} \subset K^{R_{t_{0}}}
\end{gathered}
$$

holds. Therefore, if $\psi \in R_{t_{0}}$ holds, then $T_{t_{0}}$ is a subset of $\overline{X_{\psi}^{\prime}}, \chi_{\uparrow T_{t_{0}}}$ is a continuous map from $T_{t_{0}}$ to $W_{R}$ and $\psi \circ\left(u_{\uparrow T_{t_{0}}}\right)$ is a continuous map from $T_{t_{0}}$ to $\overline{D_{r_{0}}^{n}}$ such that for any $t \in T_{t_{0}},\left(\left(\psi \circ\left(u_{\uparrow T_{t_{0}}}\right)\right)(t), t\right) \in K^{R_{t_{0}}}, \psi$ holds.

$5^{\circ}:$ For a subset $T^{\prime}$ of $T$, we define

$$
\begin{aligned}
U^{T^{\prime}}:= & U \cap \pi^{-1}\left(T^{\prime}\right)=\exp \left(\cup_{p \in u\left(T^{\prime}\right)} B_{p}^{\prime}\left(T_{\mathbb{R}}\right)\right), \\
& \left.\exp \right|_{T^{\prime}}:=\exp _{\mid \cup_{p \in u\left(T^{\prime}\right)} B_{p}^{\prime}\left(T_{\mathbb{R}}\right)} .
\end{aligned}
$$

Let $t_{0} \in T$. We examine the map $\left.\exp \right|_{T_{t_{0}}}$ and its range $U^{T_{t_{0}}}$ and the map exp $\left.\right|_{\left\{t_{0}\right\}}$ and its range $U^{\left\{t_{0}\right\}}$.

By $1^{\circ}$, there exists $\psi \in R_{t_{0}}$. In virtue of $4^{\circ}, u\left(T_{t_{0}}\right) \subset \cap_{\varphi \in R_{t_{0}}} M_{\varphi}^{\prime} \subset M_{\psi}$ holds. Now, because $\cup_{p \in u\left(T_{t_{0}}\right)} B_{p}^{\prime}\left(T_{\mathbb{R}}\right)$ is an open set of $u^{*}\left(T_{\mathbb{R}}(M)\right) \mid T_{t_{0}}\left(=T_{\mathbb{R}}(M) \mid u\left(T_{t_{0}}\right)=\cup_{p \in u\left(T_{t_{0}}\right)}\left(T_{\mathbb{R}}\right)_{p}\left(F_{p}\right)\right)$, the trivialization coordinate system

$$
\dot{p} \in u^{\star}\left(T_{\mathbb{R}}(M)\right) \mid T_{t_{0}} \mapsto\left(\left(D\left(\left.\psi\right|_{\pi(\varpi(\dot{p}))}\right)\right)_{\varpi(\dot{p})}(\dot{p}), \pi(\varpi(\dot{p}))\right) \in \mathbb{C}^{n} \times T_{t_{0}}
$$

of the vector bundle $u^{\star}\left(T_{\mathbb{R}}(M)\right) \mid T_{t_{0}}$ on $T_{t_{0}}$ maps $\cup_{p \in u\left(T_{t_{0}}\right)} B_{p}^{\prime}\left(T_{\mathbb{R}}\right)$ to an open set of $\mathbb{C}^{n} \times T_{t_{0}}$. That is, as we denote the image of the set $\cup_{p \in u\left(T_{t_{0}}\right)} B_{p}^{\prime}\left(T_{\mathbb{R}}\right)$ by $G_{t_{0}}, G_{t_{0}}$ is an open set of $\mathbb{C}^{n} \times T_{t_{0}}$. We show $G_{t_{0}} \subset U_{R_{t_{0}}, \psi, \chi_{\uparrow T_{t_{0}}}, \psi \circ\left(u_{\uparrow T_{t_{0}}}\right)}$. As $t \in T_{t_{0}}$ and $\dot{p} \in B_{u(t)}^{\prime}\left(T_{\mathbb{R}}\right)$ hold, because of $u(t) \in K^{R_{t_{0}}} \subset\left(\psi,\left.\pi\right|_{\psi}\right)^{-1}\left(\overline{D_{r_{0}}^{n}} \times \overline{X_{\psi}^{\prime}}\right)$, by Lemma 4.17,

$$
\left\|\left(D\left(\left.\psi\right|_{t}\right)\right)_{u(t)}(\dot{p})\right\|_{\mathbb{C}^{n}} \leq C_{1}^{a}\|\dot{p}\|_{u(t)}<\min \left\{\delta_{0}^{a}, \frac{\delta_{0}^{b}}{\alpha_{0}}\right\}
$$

holds. So, in virtue of $4^{\circ}$ and Lemma 4.16 ,

$$
\left((D(\psi \mid t))_{u(t)}(\dot{p}), t\right) \in U_{R_{t_{0}}, \psi, \chi_{\uparrow T_{t_{0}}}, \psi \circ\left(u_{\uparrow T_{t_{0}}}\right)}^{\prime}
$$

holds. $G_{t_{0}} \subset U_{R_{t_{0}}, \psi, \chi_{\uparrow T_{t_{0}}}, \psi \circ\left(u_{\uparrow T_{t_{0}}}\right)}^{\prime}$ holds. From the above, $G_{t_{0}}$ is an open set of $U_{R_{t_{0}}, \psi, \chi_{\uparrow T_{t_{0}}}, \psi \circ\left(u_{\uparrow T_{t_{0}}}\right)}^{\prime}$. Hence, as we set

$$
H_{t_{0}}:=\left\{\left(e^{R_{t_{0}}, \psi, \chi_{\uparrow T_{t_{0}}}, \psi \circ\left(u_{\uparrow T_{t_{0}}}\right)}(\dot{z}, t), t\right) \in D_{\frac{r_{0}+1}{2}}^{n} \times T_{t_{0}} \mid(\dot{z}, t) \in G_{t_{0}}\right\},
$$

by Lemma 4.16, $H_{t_{0}}$ is an open set of $D_{\frac{r_{0}+1}{2}}^{n} \times T_{t_{0}}$ and homeomorphically the map

$$
(\dot{z}, t) \mapsto\left(e^{R_{t_{0}}, \psi, \chi \uparrow T_{t_{0}}}, \psi \circ\left(u_{\uparrow T_{t_{0}}}\right)(\dot{z}, t), t\right)
$$

maps $G_{t_{0}}$ to $H_{t_{0}}$. Therefore, $U^{T_{t_{0}}}$ is an open set of $\pi^{-1}\left(T_{t_{0}}\right)$ and homeomorphically the map exp $\left.\right|_{T_{t_{0}}}$ maps to its domain $\cup_{p \in u\left(T_{t_{0}}\right)} B_{p}^{\prime}\left(T_{\mathbb{R}}\right)$ to its range $U^{T_{t_{0}}}$. Further, exp $\left.\right|_{\left\{t_{0}\right\}}$ is a biholomorphic map from its domain $B_{u\left(t_{0}\right)}^{\prime}\left(T_{\mathbb{R}}\right)$ to its range $U^{\left\{t_{0}\right\}}$. Also,

$$
u\left(t_{0}\right)=\left.\exp \right|_{\left\{t_{0}\right\}}\left(0_{u\left(t_{0}\right)}\right) \in U^{\left\{t_{0}\right\}}
$$

holds. Also, because for $\dot{p} \in B_{u\left(t_{0}\right)}^{\prime}\left(T_{\mathbb{R}}\right)$,

$$
\begin{gathered}
\left(D\left(\left.\exp \right|_{\left\{t_{0}\right\}}\right)\right)_{\dot{p}} \\
=\left(D\left(\left.\psi\right|_{t_{0}} ^{-1}\right)\right)_{\left(e^{R_{t_{0}}, \psi, \chi_{\uparrow T_{t_{0}}}, \psi \circ\left(u_{\uparrow T_{t_{0}}}\right)}\left(\left(D\left(\psi \mid t_{0}\right)\right)_{u\left(t_{0}\right)}(\dot{p}), t_{0}\right), t_{0}\right)} \\
\cdot\left(D_{\dot{z}} e^{R_{t_{0}}, \psi, \chi_{\uparrow T_{t_{0}}}, \psi \circ\left(u_{\uparrow T_{t_{0}}}\right)}\right)\left(\left(D\left(\left.\psi\right|_{t_{0}}\right)\right)_{u\left(t_{0}\right)}(\dot{p}), t_{0}\right) \cdot\left(D\left(\left.\psi\right|_{t_{0}}\right)\right)_{u\left(t_{0}\right)}
\end{gathered}
$$

holds, from Lemma 4.16 and Lemma 4.17,

$$
\begin{gathered}
\sup _{\dot{p} \in B_{u\left(t_{0}\right)}^{\prime}\left(T_{\mathbb{R}}\right)}\left\|\left(D\left(\left.\exp \right|_{\left\{t_{0}\right\}}\right)\right)_{\dot{p}}\right\|_{L\left(\left(T_{\mathbb{R}}\right)_{u\left(t_{0}\right)}\left(\pi^{-1}\left(\left\{t_{0}\right\}\right)\right) ;\left(T_{\mathbb{R}}\right)_{\exp \mid}\left\{t_{0}\right\}\right.}(\dot{p}) \\
\left.\left.\leq \pi^{-1}\left(\left\{t_{0}\right\}\right)\right)\right) \\
\leq C_{1}^{b} \cdot \alpha_{0} \cdot C_{1}^{a}
\end{gathered}
$$


holds.

$6^{\circ}:$ For any $t_{0} \in T, T_{t_{0}}$ is an open set of $T$ and $t_{0} \in T_{t_{0}}$ holds. Hence, from $5^{\circ}, U$ is an open set of $\pi^{-1}(T)$ and homeomorphically the map exp maps its domain $\cup_{p \in u(T)} B_{p}^{\prime}\left(T_{\mathbb{R}}\right)$ to its range $U$. Further, for any $t \in T$,

$$
u(t)=\exp \left(0_{u(t)}\right) \in U_{t}\left(:=U \cap \pi^{-1}(\{t\})\right)
$$

holds and the map exp $\left.\right|_{\{t\}}$ is a biholomorphic map from its domain $B_{u(t)}^{\prime}\left(T_{\mathbb{R}}\right)$ to its range $U_{t}$. Also,

$$
\begin{gathered}
\sup _{t \in T, \dot{p} \in B_{u(t)}^{\prime}\left(T_{\mathbb{R}}\right)}\left\|\left(D\left(\left.\exp \right|_{\{t\}}\right)\right)_{\dot{p}}\right\|_{L\left(\left(T_{\mathbb{R}}\right)_{u(t)}\left(\pi^{-1}(\{t\})\right) ;\left(T_{\mathbb{R}}\right)_{\exp \mid\{t\}}(\dot{p})\left(\pi^{-1}(\{t\})\right)\right)} \\
\leq \alpha_{0} C_{1}^{a} C_{1}^{b}<+\infty
\end{gathered}
$$

holds.

$7^{\circ}$ : For $q \in U$, we define

$$
\Psi(q):=\frac{C_{1}^{a}}{\min \left\{\delta_{0}^{a}, \frac{\delta_{0}^{b}}{\alpha_{0}}\right\}} \exp ^{-1}(q) .
$$

From $6^{\circ},(U, \Psi)$ satisfies the conditions (1), (2) and (3) of the definition.

$8^{\circ}$ : We show that the condition (4) of the definition is satisfied.

Let $0 \leq r<1$ and $\varepsilon>0$. We set

$$
\delta:=\min \left\{\delta_{2}, \frac{\delta_{0}^{b}}{C_{2}}(1-r), \frac{\min \left\{\delta_{0}^{a}, \frac{\delta_{0}^{b}}{\alpha_{0}}\right\}}{\beta_{0} C_{1}^{a} C_{1}^{b} C_{2}}(1-r), \frac{\min \left\{\delta_{0}^{a}, \frac{\delta_{0}^{b}}{\alpha_{0}}\right\}}{\beta_{0} C_{1}^{a} C_{1}^{b} C_{2}} \varepsilon\right\} .
$$

$\delta>0$ holds.

Suppose $t \in T, p \in U_{t}, q \in \pi^{-1}(\{t\}),\|\Psi(p)\|_{u(t)} \leq r$ and $d_{t}(q, p)<\delta$. We show $q \in U_{t}$ and $\| \Psi(q)-$ $\Psi(p) \|_{u(t)}<\varepsilon$. From $1^{\circ}$, there exists $\psi$ such that $\psi \in R_{t} \subset S$ holds. On the other hand,

$$
\left\|\exp ^{-1}(p)\right\|_{u(t)} \leq \frac{\min \left\{\delta_{0}^{a}, \frac{\delta_{0}^{b}}{\alpha_{0}}\right\}}{C_{1}^{a}} r
$$

holds. So, in virtue of $1^{\circ}$ and Lemma 4.17,

$$
\left\|(D(\psi \mid t))_{u(t)}\left(\exp ^{-1}(p)\right)\right\|_{\mathbb{C}^{n}} \leq \min \left\{\delta_{0}^{a}, \frac{\delta_{0}^{b}}{\alpha_{0}}\right\} r
$$

holds and for any $s \in[0,1]$,

$$
\left\|s(D(\psi \mid t))_{u(t)}\left(\exp ^{-1}(p)\right)\right\|_{\mathbb{C}^{n}}<\delta_{0}^{a}
$$

holds. Hence, in virtue of $1^{\circ}$ and Lemma 4.14,

$$
\begin{gathered}
e^{R_{t}, \psi}\left((D(\psi \mid t))_{u(t)}\left(\exp ^{-1}(p)\right), \psi(u(t)), t, \chi(t)\right) \in D_{\frac{r_{0}+1}{2}}^{n}, \\
\left\|e^{R_{t}, \psi}\left(\left(D\left(\left.\psi\right|_{t}\right)\right)_{u(t)}\left(\exp ^{-1}(p)\right), \psi(u(t)), t, \chi(t)\right)-\psi(u(t))\right\|_{\mathbb{C}^{n}} \\
=\left\|e^{R_{t}, \psi}\left((D(\psi \mid t))_{u(t)}\left(\exp ^{-1}(p)\right), \psi(u(t)), t, \chi(t)\right)-e^{R_{t}, \psi}(0, \psi(u(t)), t, \chi(t))\right\|_{\mathbb{C}^{n}} \\
\leq \alpha_{0}\left\|\left(D\left(\left.\psi\right|_{t}\right)\right)_{u(t)}\left(\exp ^{-1}(p)\right)\right\|_{\mathbb{C}^{n}} \leq \delta_{0}^{b} r
\end{gathered}
$$

hold. Now, because of

$$
\begin{gathered}
e^{R_{t}, \psi}\left((D(\psi \mid t))_{u(t)}\left(\exp ^{-1}(p)\right), \psi(u(t)), t, \chi(t)\right)=\psi\left(\exp \left(\exp ^{-1}(p)\right)\right), \\
p \in M_{\psi}, \quad \psi(p) \in D_{\frac{r_{0}+1}{2}}^{n} \\
\|\psi(p)-\psi(u(t))\|_{\mathbb{C}^{n}} \leq \delta_{0}^{b} r
\end{gathered}
$$


hold. Since from $p \in U_{t}\left(=U \cap \pi^{-1}(\{t\})\right)$ and $1^{\circ}, \pi(p)=t=\pi(u(t)) \in X_{\psi}^{\prime}$ holds, $(\psi(p), \pi(p)) \in D_{\frac{r_{0}+1}{2}}^{n} \times X_{\psi}^{\prime}$ holds. Therefore, by $d_{t}(q, p)<\delta \leq \delta_{2}$ and Lemma 4.18, $q \in M_{\psi}$ and

$$
\|\psi(q)-\psi(p)\|_{\mathbb{C}^{n}} \leq C_{2} d_{t}(q, p)
$$

hold. From (4.8) and (4.9),

$$
\begin{gathered}
\|\psi(p)-\psi(u(t))\|_{\mathbb{C}^{n}}<\delta_{0}^{b}, \\
\|\psi(q)-\psi(u(t))\|_{\mathbb{C}^{n}} \leq C_{2} d_{t}(q, p)+\delta_{0}^{b} r \\
<C_{2} \delta+\delta_{0}^{b} r \leq C_{2} \frac{\delta_{0}^{b}}{C_{2}}(1-r)+\delta_{0}^{b} r=\delta_{0}^{b}
\end{gathered}
$$

hold. So, for $s \in[0,1]$, as we set

$$
\begin{gathered}
c(s):=(1-s) \psi(p)+s \psi(q)=\psi(p)+s(\psi(q)-\psi(p)), \\
\|c(s)-\psi(u(t))\|_{\mathbb{C}^{n}}<\delta_{0}^{b}
\end{gathered}
$$

holds. Therefore, in virtue of $1^{\circ}$, Lemma 4.14 and (4.9), 


$$
\begin{gathered}
(c(s), \psi(u(t)), t, \chi(t)) \in V_{R_{t}, \psi}^{\prime}, \\
\left\|h^{R_{t}, \psi}(\psi(q), \psi(u(t)), t, \chi(t))-h^{R_{t}, \psi}(\psi(p), \psi(u(t)), t, \chi(t))\right\|_{\mathbb{C}^{n}} \\
\leq \beta_{0}\|\psi(q)-\psi(p)\|_{\mathbb{C}^{n}} \leq \beta_{0} C_{2} d_{t}(q, p)
\end{gathered}
$$

hold. Hence, further, from $p \in U_{t}=U \cap \pi^{-1}(\{t\})$,

$$
h^{R_{t}, \psi}(\psi(p), \psi(u(t)), t, \chi(t))=(D(\psi \mid t))_{u(t)}\left(\exp ^{-1}(p)\right)
$$

holds. Now, we set

$$
\dot{z}:=h^{R_{t}, \psi}(\psi(q), \psi(u(t)), t, \chi(t)) .
$$

Then,

$$
\left\|\dot{z}-(D(\psi \mid t))_{u(t)}\left(\exp ^{-1}(p)\right)\right\|_{\mathbb{C}^{n}} \leq \beta_{0} C_{2} d_{t}(q, p)
$$

holds. In virtue of $1^{\circ}$ and Lemma 4.17,

$$
\left\|(D(\psi \mid t))_{u(t)}^{-1}(\dot{z})-\exp ^{-1}(p)\right\|_{u(t)} \leq \beta_{0} C_{1}^{b} C_{2} d_{t}(q, p)
$$

holds. From (4.7) and (4.10),

$$
\begin{gathered}
\left\|(D(\psi \mid t))_{u(t)}^{-1}(\dot{z})\right\|_{u(t)} \\
\leq \beta_{0} C_{1}^{b} C_{2} d_{t}(q, p)+\frac{\min \left\{\delta_{0}^{a}, \frac{\delta_{0}^{b}}{\alpha_{0}}\right\}}{C_{1}^{a}} r \\
<\beta_{0} C_{1}^{b} C_{2} \delta+\frac{\min \left\{\delta_{0}^{a}, \frac{\delta_{0}^{b}}{\alpha_{0}}\right\}}{C_{1}^{a}} r \\
\leq \beta_{0} C_{1}^{b} C_{2} \frac{\min \left\{\delta_{0}^{a}, \frac{\delta_{0}^{b}}{\alpha_{0}}\right\}}{\beta_{0} C_{1}^{a} C_{1}^{b} C_{2}}(1-r)+\frac{\min \left\{\delta_{0}^{a}, \frac{\delta_{0}^{b}}{\alpha_{0}}\right\}}{C_{1}^{a}} r \\
=\frac{\min \left\{\delta_{0}^{a}, \frac{\delta_{0}^{b}}{\alpha_{0}}\right\}}{C_{1}^{a}}
\end{gathered}
$$

holds. That is,

$$
(D(\psi \mid t))_{u(t)}^{-1}(\dot{z}) \in B_{u(t)}^{\prime}\left(T_{\mathbb{R}}\right)
$$

holds. Therefore,

$$
\left.q=\exp \left(\left(D(\psi \mid t)_{t}\right)\right)_{u(t)}^{-1}(\dot{z})\right) \in U \cap \pi^{-1}(\{t\})=U_{t}
$$

holds. Further, from (4.10),

$$
\begin{gathered}
\|\Psi(q)-\Psi(p)\|_{u(t)} \\
=\frac{C_{1}^{a}}{\min \left\{\delta_{0}^{a}, \frac{\delta_{0}^{b}}{\alpha_{0}}\right\}}\left\|\left(D\left(\left.\psi\right|_{t}\right)\right)_{u(t)}^{-1}(\dot{z})-\exp ^{-1}(p)\right\|_{u(t)} \\
\leq \frac{\beta_{0} C_{1}^{a} C_{1}^{b} C_{2}}{\min \left\{\delta_{0}^{a}, \frac{\delta_{0}^{b}}{\alpha_{0}}\right\}} d_{t}(q, p) \\
<\frac{\beta_{0} C_{1}^{a} C_{1}^{b} C_{2}}{\min \left\{\delta_{0}^{a}, \frac{\delta_{0}^{b}}{\alpha_{0}}\right\}} \delta \\
\leq \frac{\beta_{0} C_{1}^{a} C_{1}^{b} C_{2}}{\min \left\{\delta_{0}^{a}, \frac{\delta_{0}^{b}}{\alpha_{0}}\right\}} \frac{\min \left\{\delta_{0}^{a}, \frac{\delta_{0}^{b}}{\alpha_{0}}\right\}}{\beta_{0} C_{1}^{a} C_{1}^{b} C_{2}} \varepsilon=\varepsilon
\end{gathered}
$$

holds.

Acknowledgement: The comments from the reviewers were helpful. This work was supported by JSPS KAKENHI Grant Number JP16K05245. 


\section{References}

[1] B. W. Glickfeld, The Riemann sphere of a commutative Banach algebra, Trans. Amer. Math. Soc., 134 (1968), 1-28.

[2] K. R. Goodearl, Cancellation of low-rank vector bundles, Pacific J. Math., 113 (1984), 289-302.

[3] E. Hille and R. S. Phillips, Functional analysis and semi-groups, American Mathematical Society, 1957.

[4] S. Kobayashi, Manifolds over function algebras and mapping spaces, Tohoku Math. J., 41 (1989), 263-282.

[5] K. Kodaira, Complex manifolds and deformation of complex structures, Springer-Verlag, 1986.

[6] K. Kodaira and D. C. Spencer, On deformations of complex analytic structures, Ann. of Math., 67 (1958), 328-401.

[7] E. R. Lorch, The theory of analytic functions in normed Abelian vector rings, Trans. Amer. Math. Soc., 54 (1943), 414-425.

[8] P. Manoharan, A nonlinear version of Swan's theorem, Math. Z., 209 (1992), 467-479.

[9] P. Manoharan, Generalized Swan's theorem and its application, Proc. Amer. Math. Soc., 123 (1995), 3219-3223.

[10] P. Manoharan, A characterization for spaces of sections, Proc. Amer. Math. Soc., 126 (1998), 1205-1210.

[11] A. S. Morye, Note on the Serre-Swan theorem, Math. Nachr., 286 (2013), 272-278.

[12] J. Mujica, Complex analysis in Banach spaces, North-Holland, 1986.

[13] R. G. Swan, Vector bundles and projective modules, Trans. Amer. Math. Soc., 105 (1962), 264-277.

[14] L. N. Vaserstein, Vector bundles and projective modules, Trans. Amer. Math. Soc., 294 (1986), 749-755.

[15] H. Yagisita, Holomorphic differential forms of complex manifolds on commutative Banach algebras and a few related problems, preprint.

[16] H. Yagisita, Cartan-Thullen theorem for a $\mathbb{C}^{n}$-holomorphic function and a related problem, preprint. 